\title{
Thermally active interphase inclusion in a smooth contact conditions with transversely isotropic half-spaces
}

\author{
Oleksandr Kryvyi \\ National University «Odesa Maritime Academy», 65029, Odessa, Didrikbson str., 8, Ukraine \\ krivoy-odessa@ukr.net,kryvyi-od@math.onma.edu.ua, bttp:/ /orcid.org/0000-0001-2345-6789
}

\author{
Yurii Morozov \\ Odesa National Polytechnic University, 65044, Odesa, Boulevard of Shevchenko, 1, Ukraine \\ morozov@opu.ua,morozovyu@gmail.com, bttps://orcid.org/0000-0003-4027-2353
}

\begin{abstract}
An exact solution of the stationary thermoelasticity problem is constructed for the interfacial circular absolutely rigid inclusion, while it is in the smooth contact conditions in a piecewise homogeneous transversely homogeneous space. With the help of the constructed discontinuous solution, by the method of singular integral relations, the task is reduced to a system of singular integral equations (SIE).An exact solution has been built for the specified SIE, and as a result, dependences of translational displacement of the inclusion on temperature, the resultant load, main moment and thermomechanical characteristics of transversely isotropic materials have been obtained.
\end{abstract}

KEYWORDS. Thermoelasticity problem; Interphase circular inclusion; Singular integral equations; Piecewise-homogeneous transversely isotropic space.

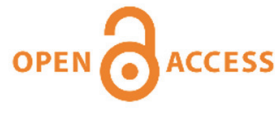

Citation: Kryvyi, O., Morozov Yu., Thermally active interphase inclusion in a smooth contact conditions with transversely isotropic half-spaces, Frattura ed Integrità Strutturale, 52 (2020) 32-50.

Received: 16.07 .2019

Accepted: 24.01 .2020

Published: 01.04.2020

Copyright: (C) 2020 This is an open access article under the terms of the CC-BY 4.0, which permits unrestricted use, distribution, and reproduction in any medium, provided the original author and source are credited.

\section{INTRODUCTION}

T he modern construction is widely using composite anisotropic materials and heterogeneous structures that contain constructive or technological interphase heat-active inclusions (defects, fibers, reinforcement elements) and are under conditions of power and temperature loading with various types of contact interaction with the medium.

These inclusions are significant stress concentrators and can lead to structural failure as well as to a the destruction of structures. At the same time stress concentration is significantly affected as a form of contact interaction of the inclusion with the medium so are the ratio of thermoelastic constants of the matrix.

Such problems arise [1,2], particularly, in geophysics, during the operation of nuclear power plants, in welded bridge structures and when using modern reinforced materials.

One of the methods of repairing damaged structures of long-term operation is injection technologies [3], according to which the damaged areas of concrete and reinforced concrete structures and structures are filled with special materials, turning over time into rigid heat-emitting inclusions that may have different contact with the matrix. 
Therefore, an important scientific and technical task for of predicting the possible destruction of structures during their operation and design is a study of the stress-strain state of bodies in the presence of interphase heat-emitting or heatinsulated inclusions in anisotropic piecewise homogeneous media.

In particular, the study of the effects on deformation and the distribution of stresses around the specified inclusions of thermoelastic parameters of anisotropic media and the type of interaction contact of inclusions with the medium.

In the general this kind of finite bodies problems in the presence of many inclusions, can have only a numerical solution, . However, the presence of exact solutions for single inclusions allows not only to study the stress concentration around the defect, but also improve the efficiency of numerical methods.

Concentration of tensions near defects such as cracks or inclusions in the composite media have been investigated by many authors. In particular, in [5] non-axisymmetric problems on various interfacial defects in a composite isotropic space are reduced to systems of two-dimensional singular integral equations (SIE), and for circular defects a method for their exact solutions is proposed. In [6], the axisymmetric problem of the theory of elasticity for a rigid circular inclusion located at the interface between two perfectly connected dissimilar isotropic elastic half-spaces is considered, the solution for which was obtained using the Hankel transform.

In papers [7-9], using the method of potential and the Fourier transform, the problems of stationary thermoelasticity for absolutely rigid inclusion in unbounded isotropic space, and in [6] in transversely isotropic space under the action of a heat flux, are reduced to a system of related two-dimensional singular integral equations for jumps of stress.

In $[1,2]$, consider stationary thermal elasticity problems for the body with a thermally permeable disk inclusion, between the surfaces where there is an imperfect thermal contact, as well as with a thin heat-active disk inclusion. The posed problems are reduced to hypersingular integral equations of the first and second kind, for which an exact solution has been obtained.

In works [11-14], problems on of nonaxisymmetric interphase defects such as cracks or absolutely rigid inclusions, for different types of contact interaction (full coupling, smooth contact, mixed conditions) with various transversely isotropic half-spaces using the method of singular integral relations (SIR) [15] are being reduced to two-dimensional SIE systems. A method of constructing exact solutions of these SIA systems for circular defects has been proposed, which has allowed to determine the singularity of the stress and displacement fields around the cracks and inclusions under arbitrary load.

An analysis of well-known publications shows that nonaxisymmetric problems of thermoelasticity for piecewise homogeneous transversely isotropic media in the presence of interfacial defects are not well-studied.have not been extencively studied. Especially it concerns a smooth (sliding) contact of the inclusion with the medium, despite the importance of such a solution [16].

In this paper, for the first time, we consider the non-axisymmetric stationary thermoelasticity problem for a composite transversely isotropic space containing a heat radiating circular inclusion having a smooth contact with the medium. The problem, using the SIR method, is reduced to the SIE system. An exact solution to the indicated SIE system was obtained, which made it possible to study the fields of stresses, strains, and temperatures around the inclusion.

\section{FORMULATION OF THE PROBLEM}

T et in the plane $z=0$ everywhere except the circular area $\Omega:\left\{\sqrt{x^{2}+y^{2}} \leq a\right\}$, have the perfect contact of two different transversely isotropic half-spaces. For stress tensor components: $\sigma_{z}, \tau_{y z}, \tau_{x z}$, vector of movements $u, v, w$, temperature $T$ and heat flux $q_{z}$, we introduce the notation:

$$
\left\{\zeta_{k}^{ \pm}\right\}^{8}=\left.\left\{v_{k}\right\}^{8}\right|_{z= \pm 0}, \mathbf{v}=\left\{v_{k}(x, y, z)\right\}_{k=1,8}=\left\{\sigma_{z}, \tau_{y z}, \tau_{x z}, u, v, w, T, q_{z}\right\}
$$

In view of the notation (1), the conditions for perfect contact of various transversely isotropic half-spaces outside the area $\Omega$ can be written as

$$
\chi_{k}^{-}(x, y)=0,(k=\overline{1,7}), \lambda_{3}^{+} \partial_{2} \zeta_{7}(x, y,+0)=\lambda_{3}^{-} \partial_{2} \zeta_{7}(x, y,-0),(x, y) \notin \Omega,
$$

where

$$
\chi_{k}^{ \pm}=\left\langle\zeta_{k}(x, y)\right\rangle^{ \pm}=\zeta_{k}^{+}(x, y) \pm \zeta_{k}^{-}(x, y), \quad k=\overline{1,8}
$$


$\lambda_{3}^{ \pm}-$coefficient of thermal conductivity along the axis $Z$, respectively, for the upper and lower half-space.

The area $\Omega$ contains a heat-active absolutely rigid inclusion (Fig. 1), being in smooth contact with the medium. The shape of inclusion faces is described by functions $\vartheta_{0}^{ \pm}(x, y),(x, y) \in \Omega$, thus $\vartheta_{0}^{+}(x, y)>0, \vartheta_{0}^{-}(x, y)<0$, except for that we shall consider satisfied a condition:

$$
\left|\vartheta^{-}(x, y)\right|<0.25 a,\left(\vartheta^{ \pm}(x, y)=\vartheta_{0}^{+}(x, y) \pm \vartheta_{0}^{-}(x, y)\right)
$$

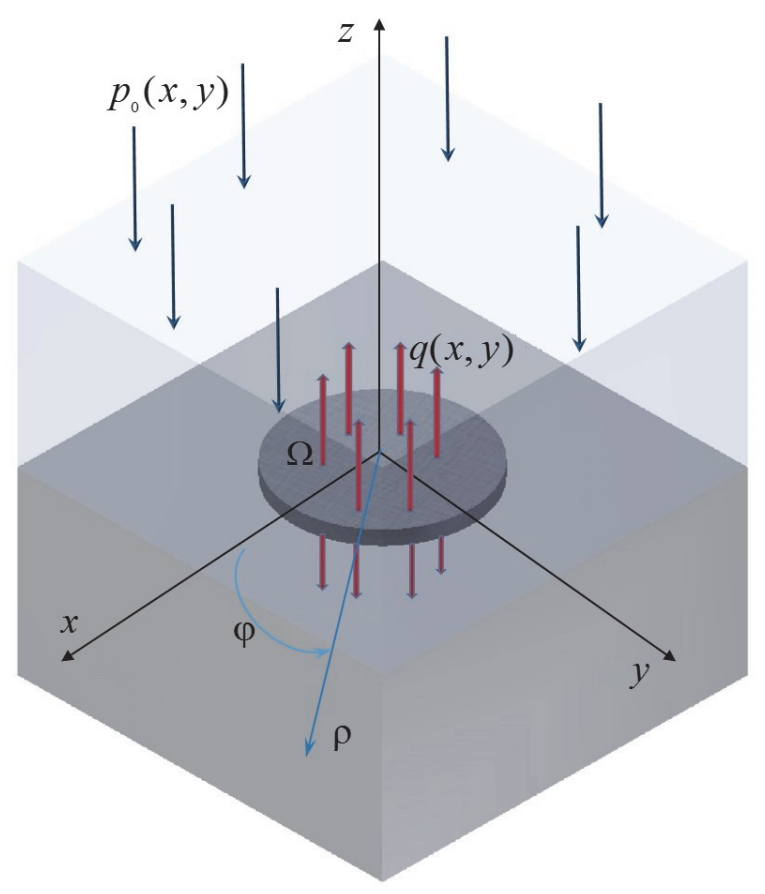

Figure 1: CAPTION IS MISSING.

If condition (4) is satisfied, the inclusion can be considered thin, and since it is absolutely rigid, the effect of its faces on half-spaces (boundary conditions) can be carried on the planes: $z= \pm 0,(x, y) \in \Omega$.. This approach is some simplification of the mechanical model, however, as shown by the comparison with the solution of individual problems in the general arrangement [17], the error is not significant and tends to zero with reduction of the inclusion thickness. At the same time, with this approach, the mathematical model of the problem is greatly simplified, which allows to obtain its exact solution. This approach, in particular, was applied in [18] to study the dependence on the form of inclusion of the stress concentration in the neighborhood of interfacial defects in a composite anisotropic medium.

Let's assume that on infinity to the environment along the axis $z$ normal compressive load applied $p_{0}(x, y)$ which on inclusion leads to the resultant force $P_{z}$ and the main moments $M_{x}, M_{y}$ and on the inclusion the heat flow is se $q(x, y)$. As a result of such a force and thermal actions, the positions of the inclusion faces will be described by the functions

$$
\zeta_{6}^{ \pm}=\zeta_{6}^{0}+\vartheta_{0}^{ \pm}(x, y), \quad \zeta_{6}^{0}=\delta_{z}+\varphi_{y} y+\varphi_{x} x,(x, y) \in \Omega,
$$

where $\delta_{z}, \varphi_{x, y}$-translational displacements and turning angles of the inclusion around the corresponding axes, for determine of which are use force and moment equilibrium equations

$$
\iint_{\Omega} \chi_{1}(x, y) d x d y=P_{z}, \iint_{\Omega}\left(\begin{array}{c}
x \\
y
\end{array}\right) \chi_{1}(x, y) d x d y=\left(\begin{array}{c}
M_{y} \\
M_{x}
\end{array}\right) .
$$


With this formulation of the problem, the tangent stresses are equal to zero on the inclusion faces, normal displacements and heat flux are also specified. According to the notation (1) and (3), we formulate these conditions

$$
\chi_{k}^{ \pm}(x, y)=0, k=2,3, \chi_{6}^{ \pm}(x, y)=\vartheta^{ \pm}(x, y)+(1 \pm 1) \zeta_{6}^{0}, \zeta_{8}^{ \pm}(x, y, \pm 0)=q(x, y), \quad(x, y) \in \Omega .
$$

Unknown in the area $\Omega$ for the task will be jumps of normal stresses, tangential displacements and temperature:

$$
\chi_{k}^{-}(x, y),(k=1,4,5,7)
$$

\section{REDUCTION OF THE PROBLEM TO A TWO-DIMENSIONAL SYSTEM OF SIE AND CONSTRUCTION OF ITS EXACT SOLUTION.}

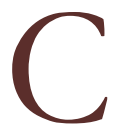
onditions (7) make it possible to use the approach proposed in [11-14] to reduce the problem to a twodimensional system of SIE relatively unknown jumps (8). This approach is based on the generalized functions of slow growth constructed in [15] in the space $\mathfrak{I}^{\prime}\left(R^{3}\right)$ discontinuous solutions and SIS for a piecewise homogeneous transversely isotropic space. It should be emphasized that solutions were built in space of $\mathfrak{J}^{\prime}\left(R^{3}\right)$ to strictly justify all constructions and prove the existence of solutions in the respective classes.

Following [15], an SIS (A.11) was obtained in the appendix, relating jumps and sums (3) of the thermoelastic characteristics of the medium. The second, third, fourth and sixth equality from (A.11), as well as conditions (7), allowed us to obtain relatively about unknown $\chi_{1}^{-}, u^{-}=\chi_{4}^{-}+i \chi_{5}^{-}, \chi_{7}^{-}$the next system of four two-dimensional SIE

$$
\begin{aligned}
& q_{21} \mathrm{DK}\left[\chi_{1}^{-}\right]+\frac{q_{23}^{-}}{2} \overline{\mathrm{D}}^{2} \mathrm{~K}\left[u^{-}\right]+\frac{q_{23}^{+}}{2} \mathrm{D} \overline{\mathrm{D}} \mathrm{K}\left[\bar{u}^{-}\right]=-q_{24} \mathrm{D} \chi_{6}^{-}-q_{25} \mathrm{DK}\left[\chi_{7}^{-}\right], \\
& q_{21} \overline{\mathrm{D}} \mathrm{K}\left[\chi_{1}^{-}\right]+\frac{q_{23}^{-}}{2} \mathrm{D} \overline{\mathrm{D}} \mathrm{K}\left[\bar{u}^{-}\right]+\frac{q_{23}^{+}}{2} \overline{\mathrm{D}}^{2} \mathrm{~K}\left[u^{-}\right]=-q_{24} \overline{\mathrm{D}} \chi_{6}^{-}-q_{25} \overline{\mathrm{D}} \mathrm{K}\left[\chi_{7}^{-}\right], \\
& q_{41} \mathrm{~K}\left[\chi_{1}^{-}\right]+\frac{q_{43}}{2}\left[\overline{\mathrm{D}} \mathrm{K}\left[u^{-}\right]+\mathrm{DK}\left[\bar{u}^{-}\right]\right]=\chi_{6}^{+}-q_{44} \chi_{6}^{-}-q_{45} \mathrm{~K}\left[\chi_{7}^{-}\right], \\
& -\frac{q_{65}}{2 \pi} \mathrm{D} \overline{\mathrm{D}} \mathrm{K}\left[\chi_{7}^{-}\right]=\chi_{8}^{+}(x, y),(x, y) \in \Omega .
\end{aligned}
$$

Here, operators $\mathrm{D}$ and $\mathrm{K}$ defined by relations (A.10) and (A.12).

Let's consider for definiteness, that a heat flux in the area $\Omega$ of changes under the linear law:

$$
q(x, y)=q_{0}\left(1+d_{10} x+d_{01} y\right)
$$

We obtain the exact solution to system (9) using the approach described in [11-14]. To do this, we turn to the thermoelastic characteristics of space in cylindrical coordinates $(\rho, \varphi, z)$, jumps and the sums at transition through a plane $z=0$ we shall designate as follows: $\left\{\left\langle\sigma_{z}\right\rangle^{ \pm},\left\langle\tau_{z \varphi}\right\rangle^{ \pm},\left\langle\tau_{z \rho}\right\rangle^{ \pm},\left\langle u_{\rho}\right\rangle^{ \pm},\left\langle u_{\varphi}\right\rangle^{ \pm},\langle w\rangle^{ \pm},\langle T\rangle^{ \pm},\langle q\rangle^{ \pm}\right\}=\left\{\tilde{v}_{k}^{ \pm}(\rho, \varphi)\right\}^{8}$. In polar coordinate system $(\rho, \varphi)$ instead of unknown functions $(8)$ we will enter new unknown functions:

$$
v_{1}^{-}(r, \varphi)=\chi_{1}^{-}(\rho \cos \varphi, \rho \sin \varphi), v_{3}(r, \varphi)=e^{-i \varphi} u^{-}(\rho \cos \varphi, \rho \sin \varphi), v_{5}^{-}(r, \varphi)=\chi_{7}^{-}(\rho \cos \varphi, \rho \sin \varphi) .
$$

functions (11) are searched in the form of

$$
v_{j}^{ \pm}(\varrho, \varphi)=\sum_{n=-\infty}^{\infty} \mathrm{V}_{n}^{j, \pm}(\varrho) e^{i n \varphi}
$$




$$
\mathrm{V}_{n}^{j,-}(\rho)=\Phi_{n}\left[v_{j}^{-}\right] \equiv \frac{1}{2 \pi} \int_{-\pi}^{\pi} v_{j}^{-}(\varrho, \varphi) e^{-i n \varphi} d \varphi, j=1,3,5, \mathrm{~V}_{-n}^{3,-}(\rho)=\overline{\mathrm{V}}_{n}^{3,-}(\rho), \overline{\mathrm{V}}_{n}^{3,-}(\rho)=\Phi_{n}\left[\bar{\nu}_{3}^{-}\right]
$$

For determination of coefficientв $\mathrm{V}_{n}^{j,-}(\varrho) \quad(n=0, \pm 1, \pm 2, \pm 3, \ldots)$ in expansions (12), we move on in system (9) to polar coordinates and apply the finite Fourier transform. Then, taking into account formulas [19]

$$
\begin{aligned}
& \frac{1}{\sqrt{\rho^{2}-2 \rho \rho_{*} \cos (\varphi-\varsigma)+\rho_{*}^{2}}}, \quad=\sum_{k=-\infty}^{\infty} e^{i k(\varphi-\varsigma)} \int_{0}^{\infty} J_{k}(t \rho) J_{k}\left(t \rho_{*}\right) d t, \\
& \int_{-\pi}^{\pi} \frac{e^{-i \varphi m} d \varphi}{\rho-\rho_{*} e^{ \pm i \varphi}}= \pm \mathrm{W}_{m \pm 1, m}^{0}\left(\rho, \rho_{*}\right), \mathrm{W}_{k n}^{0}\left(\rho, \rho_{*}\right)=\int_{0}^{\infty} J_{k}(\rho t) J_{n}\left(\rho_{*} t\right) d t,
\end{aligned}
$$

of representation (12), and the convolution theorem, after simple transformations, we obtain the following system of integral equations

$$
\mathbf{B} \mathrm{W}_{n n}[\overrightarrow{\mathrm{U}}]=\mathbf{G}_{n}, \mathrm{~W}_{n+1, n+1}\left[\mathrm{U}_{4 n}\right]=\mathrm{G}_{4 n}, 0<\rho<a, n=0, \pm 1, \pm 2, \ldots,
$$

Here we use the notation

$$
\begin{aligned}
& \overrightarrow{\mathrm{U}}=\left\{\mathrm{U}_{1 n}, \mathrm{U}_{2 n}, \mathrm{U}_{3 n}\right\}, \mathrm{U}_{1 n}=V_{n}^{1,-}(\rho), \mathrm{U}_{j n}=0.5\left(\mathrm{U}_{3 n}^{*}+(-1)^{j} \overline{\mathrm{U}}_{3 n}^{*}\right), j=2,3, \\
& \mathrm{U}_{3 n}^{*}(\rho)=\rho^{-n-1}\left[\rho^{n+1} \mathrm{~V}_{n}^{3,-}(\rho)\right]^{\prime}, \overline{\mathrm{U}}_{3 n}^{*}(\rho)=\rho^{n-1}\left[\rho^{-n+1} \overline{\mathrm{V}}_{-n}^{3,-}(\rho)\right]^{\prime}, \mathrm{U}_{4 n}=\rho^{n}\left[\rho^{-n} \mathrm{~V}_{n}^{5,-}(\rho)\right]^{\prime} \\
& \mathrm{G}_{1 n}=0.5 \tilde{c}_{n}^{+} \rho^{n}-q_{24} F_{n}^{-}(\rho)-q_{25} \mathrm{~W}_{n, n}\left[\mathrm{~V}_{n}^{5,-}\right], \mathrm{G}_{3 n}=\frac{0.5 \tilde{c}_{n}^{-}}{\tilde{q}_{23}^{-}} \rho^{n}, \tilde{c}_{n}^{ \pm}=c_{n} \pm \bar{c}_{0} \delta_{0, n}, \\
& \mathrm{G}_{2 n}=F_{n}^{+}(\rho)-q_{44} F_{n}^{-}(\rho)+2 \delta_{3} \delta_{0 n}+\bar{\phi}_{12} \rho \delta_{1 n}-q_{45} \mathrm{~W}_{n, n}\left[\mathrm{~V}_{n}^{5,-}\right], F_{n}^{ \pm}(\rho)=\Phi_{n}\left[\vartheta^{ \pm}\right], \\
& \left.\mathrm{G}_{4 n}=\frac{q_{0}}{q_{65}}\left\{\frac{d_{00}}{2} \rho \delta_{0 n}+\frac{\rho^{2}}{4}\left[d_{10}\left(\delta_{1, n}+\delta_{-1, n}\right)\right)-i d_{01}\left(\delta_{1, n}-\delta_{-1, n}\right)\right]\right\}, \mathrm{W}_{n, n}[f]=\int_{0}^{a} f\left(\rho_{*}\right) \mathrm{W}_{k n}^{0}\left(\rho, \rho_{*}\right) \rho_{*} d \rho_{*}, \\
& \mathbf{B}=\left\{b_{j m}\right\}^{3}=\left(\begin{array}{ccc}
2 q_{21} & \tilde{q}_{23}^{+} & 0 \\
q_{41} & q_{43} & 0 \\
0 & 0 & 1
\end{array}\right), \Delta_{2}=\left|\begin{array}{cc}
2 q_{21} & \tilde{q}_{23}^{+} \\
q_{41} & q_{43}
\end{array}\right|=2 q_{21} q_{43}-q_{41} \tilde{q}_{23}^{+}, \tilde{q}_{23}^{ \pm}=\left(q_{23}^{-} \pm q_{23}^{+}\right), \mathbf{B}^{-1}=\left\{b_{j m}^{*}\right\}^{3}
\end{aligned}
$$

$\delta_{j, n}-$ Kronecker symbol; $c_{n}-$ constants that are determined from conditions

$$
\int_{0}^{a} U_{3 n}^{*}(\rho) \rho^{n+1} d \rho=0
$$

For the operator $\mathrm{W}_{l, l}$ there is an inverse [19], which is an isomorphism in $\mathfrak{I}^{\prime}\left(R^{3}\right)$ and allows the representation

$$
\mathrm{W}_{l, l}^{*}[f(\rho)]=-\frac{2}{\pi \rho^{1-l}} \partial_{\rho} \int_{\rho}^{a} \frac{t^{1-2 l} d t}{\left(t^{2}-\rho^{2}\right)^{1 / 2}} \frac{d}{d t} \int_{0}^{t} \frac{\tau^{1+l} f(\tau)}{\left(t^{2}-\tau^{2}\right)^{1 / 2}} d \tau
$$


The latter makes it possible, after reducing the matrix to a diagonal form, to obtain a solution to system (13):

$$
\begin{aligned}
\mathrm{U}_{j n}(\rho)= & \frac{2}{\pi \sqrt{a^{2}-\rho^{2}}}\left\{b_{j 1}^{*} 0.5 \tilde{c}_{n}^{+} \kappa_{n} \rho^{n}+b_{j 2}^{*}\left(\bar{\phi}_{12} \rho \delta_{1 n}+2 \delta_{3} \delta_{0 n}\right)\right\}+ \\
& +\mathrm{W}_{n, n}^{*}\left[b_{j 2}^{*} F_{n}^{+}(\rho)-s_{j 1} F_{n}^{-}(\rho)\right]-\tilde{s}_{j 1} \mathrm{~V}_{n}^{5,-}(\rho), j=1,2 ; \\
\mathrm{U}_{3 n}(\rho)= & \frac{\tilde{c}_{n}^{-} \kappa_{n}}{\pi \tilde{q}_{23}^{-}} \frac{\rho^{n}}{\sqrt{a^{2}-\rho^{2}}}, \\
\mathrm{U}_{4 n}(\rho)= & \left.\frac{q_{0}}{q_{65}}\left\{2 d_{00} \frac{\rho}{\sqrt{a^{2}-\rho^{2}}} \delta_{0 n}+\frac{4}{3} \frac{\rho^{2}}{\sqrt{a^{2}-\rho^{2}}}\left[d_{10}\left(\delta_{1 n}+\delta_{-1 n}\right)\right)-i d_{01}\left(\delta_{1 n}-\delta_{-1 n}\right)\right]\right\}
\end{aligned}
$$

where

$$
\begin{aligned}
& \kappa_{n}=\frac{(2 n+1)(2 n) ! !}{(2 n+1) ! !}, s_{j 1}=b_{j 1}^{*} q_{24}+b_{j 2}^{*} q_{44}, \tilde{s}_{j 1}=b_{j 1}^{*} q_{25}+b_{j 2}^{*} q_{45} . \\
& \left.\mathrm{V}_{n}^{5,-}(\rho)=\frac{q_{0}}{q_{65}}\left\{2 d_{00} \sqrt{a^{2}-\rho^{2}} \delta_{0, n}+\frac{4}{3} \rho \sqrt{a^{2}-\rho^{2}}\left[d_{10}\left(\delta_{1, n}+\delta_{-1, n}\right)\right)-i d_{01}\left(\delta_{1, n}-\delta_{-1, n}\right)\right]\right\}
\end{aligned}
$$

Equality $\mathrm{W}_{n, n}(\rho, \varsigma)=\theta(\rho-\varsigma) \varsigma^{n} \rho^{-n-1}$ which is true in $\mathfrak{J}^{\prime}\left(R^{3}\right)$, and representation (17) allow to write expressions for the coefficients in the expansion (12) so $(n=0, \pm 1, \pm 2, \ldots, 0<\rho<a)$

$$
\mathrm{V}_{n}^{1,-}(\rho)=\mathrm{U}_{1 n}, \mathrm{~V}_{n}^{3,-}(\rho)=\mathrm{W}_{n+1, n}\left[\mathrm{U}_{2 n}+\mathrm{U}_{3 n}\right], \overline{\mathrm{V}}_{n}^{3,-}(\rho)=-\mathrm{W}_{n-1, n}\left[\mathrm{U}_{2 n}-\mathrm{U}_{3 n}\right]
$$

The number of compounds in the decomposition (12) depends both on the heat flux on it and on the form of inclusion, and is generally limited. In particular, for the axial symmetric inclusion $\vartheta_{0}^{ \pm}(x, y)=\vartheta_{0}\left(x^{2}+y^{2}\right)$, $F_{n}^{ \pm}(\rho)=\Phi_{n}\left[\vartheta^{ \pm}\right]=\delta_{0, n} F^{ \pm}(\rho)$ and heat flux (10), three components will remain in decomposition (12), $n=-1,0,1$. The jumps in normal stresses and tangential displacements in this case can be represented as follows:

$$
\begin{aligned}
\left\langle\sigma_{z}\right\rangle^{-}= & \frac{m_{0}+m_{1}(\varphi) \rho}{\sqrt{a^{2}-\rho^{2}}}+\frac{s_{11}}{\rho}\left(L\left[F_{0}^{-}(\rho)\right]\right)_{\rho}^{\prime}-\tilde{s}_{11} v_{5}^{-}(\rho, \varphi), \\
\left\langle u_{\rho}\right\rangle^{-}= & \frac{2 s_{21}}{\pi \rho}\left\{L\left[F_{n}^{-}(\rho)\right]-f_{*} \sqrt{a^{2}-\rho^{2}}\right\}+ \\
& +\sqrt{a^{2}-\rho^{2}}\left\{q_{0} \operatorname{Re}\left(d e^{i \varphi}\right)\left[m_{2}\left(5 a^{2}\left(a^{2}-\rho^{2}\right)-3 \rho^{2}\right)-m_{3} a^{2}\right]-m_{4} \operatorname{Re}\left(\varphi_{x y} e^{-i \varphi}\right)\right\}, \\
\left\langle u_{\varphi}\right\rangle^{-}= & \sqrt{a^{2}-\rho^{2}}\left\{q_{0} \operatorname{Im}\left(d e^{i \varphi}\right)\left(m_{3} a^{2}-m_{2}\left(3 \rho^{2}+5 a^{2}\left(a^{2}-\rho^{2}\right)\right)-m_{4} \operatorname{Im}\left(\varphi_{x y} e^{-i \varphi}\right)\right\} .\right. \\
\langle T\rangle^{-}= & \frac{q_{0}}{q_{65}}\left\{2 d_{00} \sqrt{a^{2}-\rho^{2}}+\frac{8}{3}\left(d_{10} \cos \varphi+d_{01} \sin \varphi\right) \rho \sqrt{a^{2}-\rho^{2}}\right\}
\end{aligned}
$$

Here we use the notation

$$
\begin{aligned}
& d=d_{10}-i d_{01}, M_{x y}=M_{y}-i M_{x}, \varphi_{x y}=\varphi_{y}-i \varphi_{x}, \\
& f_{*}=\frac{1}{a} \int_{0}^{a} \frac{t F_{0}^{-}(t) d t}{\sqrt{a^{2}-t^{2}}}, L\left[F_{0}^{-}(\rho)\right]=\int_{\rho}^{a} \frac{t}{\sqrt{t^{2}-\rho^{2}}} d t \frac{d}{d t} \int_{0}^{t} \frac{\tau F_{0}^{-}(\tau)}{\sqrt{t^{2}-\tau^{2}}} d \tau,
\end{aligned}
$$




$$
\begin{aligned}
& m_{0}=\frac{P_{z}}{2 \pi}-\frac{2 f_{*}}{\pi} s_{11}+q_{0} \frac{4 a^{2}}{3} \frac{d_{00}}{q_{65}} \tilde{s}_{11}, m_{1}(\varphi)=\frac{3}{4 a^{3}} \operatorname{Re}\left(M_{x y} e^{i \varphi}\right)+\frac{4 a^{2}}{15} \frac{q_{0} \tilde{s}_{11}}{q_{65}} \operatorname{Re}\left(d e^{i \varphi}\right), \\
& m_{2}=\frac{4}{45} \frac{\tilde{s}_{21}}{q_{65}}, m_{3}=m_{2} \frac{\left(b_{21}^{*}-g_{1}\right)}{2\left(b_{21}^{*}+g_{1}\right)}, m_{4}=\frac{4 g_{1} b_{22}^{*}}{\pi\left(b_{21}^{*}+g_{1}\right)}, g_{1}=\frac{1}{\tilde{q}_{23}^{-}} .
\end{aligned}
$$

In the case of a penny-shaped inclusion of thickness $b$

$$
\begin{aligned}
& \left\langle\sigma_{z}\right\rangle^{-}=\frac{m_{0}+m_{1}(\varphi) \rho}{\sqrt{a^{2}-\rho^{2}}}-\frac{s_{11}}{\rho} \frac{b}{\sqrt{a^{2}-\rho^{2}}}+\tilde{s}_{11} v_{5}^{-}(\rho, \varphi), \\
& \left\langle u_{\rho}\right\rangle^{-}=\frac{2 b s_{21}}{\pi \rho} \sqrt{a^{2}-\rho^{2}}(1-a)+\sqrt{a^{2}-\rho^{2}}\left\{q_{0} \operatorname{Re}\left(d e^{i \varphi}\right)\left[m_{2}\left(5 a^{2}\left(a^{2}-\rho^{2}\right)-3 \rho^{2}\right)-m_{3} a^{2}\right]-m_{4} \operatorname{Re}\left(\varphi_{x y} e^{-i \varphi}\right)\right\}, \\
& \left\langle u_{\varphi}\right\rangle^{-}=\sqrt{a^{2}-\rho^{2}}\left\{q_{0} \operatorname{Im}\left(d e^{i \varphi}\right)\left(m_{3} a^{2}-m_{2}\left(3 \rho^{2}+5 a^{2}\left(a^{2}-\rho^{2}\right)\right)-m_{4} \operatorname{Im}\left(\varphi_{x y} e^{-i \varphi}\right)\right\} .\right. \\
& \langle T\rangle^{-}=\frac{q_{0}}{q_{65}}\left\{2 d_{00} \sqrt{a^{2}-\rho^{2}}+\frac{8}{3}\left(d_{10} \cos \varphi+d_{01} \sin \varphi\right) \rho \sqrt{a^{2}-\rho^{2}}\right\}
\end{aligned}
$$

For $\delta_{z}, \varphi_{x}, \varphi_{y}$ using conditions (6), the following expressions are obtained

$$
\delta_{z}=-\frac{P_{z} b_{21}}{8 a}-f_{*} m_{5}+\pi a^{2} q_{0} d_{00} m_{6}, \varphi_{y}=\frac{M_{y}}{a^{3}} m_{7}-\pi a^{2} q_{0} d_{10} m_{8}, \varphi_{x}=\frac{M_{x}}{a^{3}} m_{7}-\pi a^{2} q_{0} d_{01} m_{8},
$$

where

$$
\begin{aligned}
& m_{5}=\frac{\Delta_{2}}{2}\left(s_{11} b_{21}^{*}+b_{11}^{*} s_{21}\right), m_{6}=\frac{\Delta_{2}\left(\tilde{s}_{11} b_{21}^{*}-b_{11}^{*} \tilde{s}_{21}\right)}{6 q_{65}}, \\
& m_{7}=\frac{3\left(b_{21}^{*}+g_{1}\right)}{4\left(b_{12}^{*} b_{21}^{*}+b_{12}^{*} g_{1}-b_{11}^{*} b_{22}^{*}\right)}, m_{8}=\frac{4}{15} \frac{m_{7}}{q_{65}} \frac{b_{11}^{*} \tilde{s}_{21}-\left(b_{21}^{*}+g_{1}\right) \tilde{s}_{11}}{\left(b_{21}^{*}+g_{1}\right)} .
\end{aligned}
$$

Note that the exact solutions obtained are rigorously justified using the theory of generalized functions and the Riemann boundary value problem. The reliability of the results obtained also confirms their coincidence with the results of [20], in which a particular case of this problem for a homogeneous transversely isotropic medium is considered.

\section{NUMERICAL RESULTS AND THEIR ANALYSIS.}

$\mathrm{U}$ sing the solution obtained, we investigate the behavior the jump of normal stresses $\left\langle\sigma_{z}\right\rangle^{-}$in the neighborhood of inclusion. We assume that the load applied to the inclusion is linearly distributed, and in polar coordinates has the form: $p_{0}(\rho, \varphi)=P_{0}\left(1+\rho\left(s_{01} \sin (\varphi)+s_{10} \cos (\varphi)\right)\right.$, in this case, the resulting force and moments allow representations: $P_{z}=\pi a^{2} P_{0}, M_{y}=0.25 \pi a^{4} P_{0} s_{10}, M_{x}=0.25 \pi a^{4} P_{0} s_{01}$. Calculations were performed for the combination of materials. Cadmium (material $m 1$ ), Magnesium (material $m 2$ ), $\mathrm{Al}_{2} \mathrm{O}_{3}$ (material $m 3$ ), $\mathrm{Zn}$ (material $m 4$ ) Tab. 1 shows the values of thermoelastic constants of these materials.

Calculations were performed with $d_{01}=1 / 3, d_{10}=1 / 7, s_{01}=1 / 5, s_{10}=1 / 7, a=1$, for different values $q_{0}, P_{0}$, Figs. 2-13, for the combination of materials $\mathrm{m} 1$ (upper half space), $-\mathrm{m} 2$ (lower half space), Figs. 14-19, for the combination $\mathrm{m} 3-$ m4. Figs. 2, 4, 6, 8, 10, 12 shows the dependences $\left\langle\sigma_{z}\right\rangle^{-}$on the polar angle $\varphi$ or different values of the polar radius $\rho$,

Figs. 3, 5, 7, 9, 11, 13 show dependencies $\left\langle\sigma_{z}\right\rangle^{-}$on the polar radius $\rho$ or different values of the polar angle. In addition, 
in Figs. 2, 3, the indicated dependences were obtained for $P_{0}=0, q_{0}=70$; in Figs. 4, 5, 14, 15 with $P_{0}=0.5, q_{0}=70$; in Figs. 6, 7 with $P_{0}=0.7, q_{0}=70$; in Figs. 8, 9, 16, 17 with $P_{0}=1, q_{0}=70$; in Figs. 10, 11, with $P_{0}=0, q_{0}=150$; in Figs. $12,13,18,19$ with $P_{0}=1, q_{0}=150$..

\begin{tabular}{ccccccccccc}
\hline & \multicolumn{3}{c}{$c_{i j} \cdot 10^{-11} \mathrm{~N} / \mathrm{m}^{2}$} & \multicolumn{3}{c}{$\alpha_{j} \cdot 10^{5}\left(C^{0}\right)^{-1}$} & \multicolumn{2}{c}{$\lambda_{j} W / m C^{0}$} \\
material & $c_{11}$ & $c_{12}$ & $c_{13}$ & $c_{33}$ & $c_{44}$ & $c_{55}$ & $\alpha_{1}=\alpha_{2}$ & $\alpha_{3}$ & $\lambda_{1}=\lambda_{2}$ & $\lambda_{3}$ \\
$m 1$ & 1.08 & 0.389 & 0.375 & 0.46 & 0.156 & 0.156 & 54 & 20.2 & 93 & 94 \\
$m 2$ & 0.5952 & 0.256 & 0.214 & 0.6147 & 0.1647 & 0.1647 & 27.7 & 20.2 & 159 & 160 \\
$m 3$ & 4.6 & 1.74 & 1.27 & 5.095 & 1.269 & 1.269 & 77 & 85 & 2.6 & 3.5 \\
$m 4$ & 1.581 & 0.314 & 0.474 & 0.614 & 0.4 & 0.4 & 6.43 & 1.6 & 119 & 119 \\
\hline
\end{tabular}

Table 1: Material properties of transversely isotropic materials.

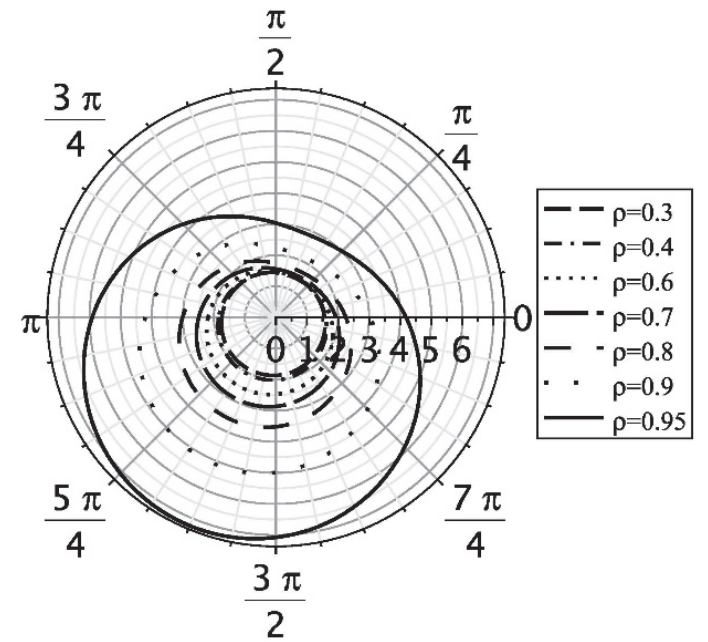

Figure 2: $P_{0}=0, q_{0}=70$

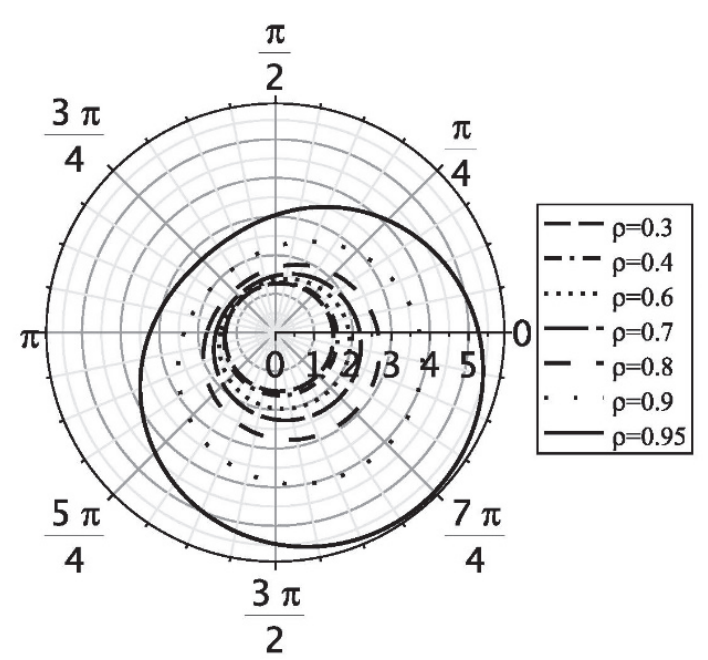

Figure 4: $P_{0}=0.5, q_{0}=70$

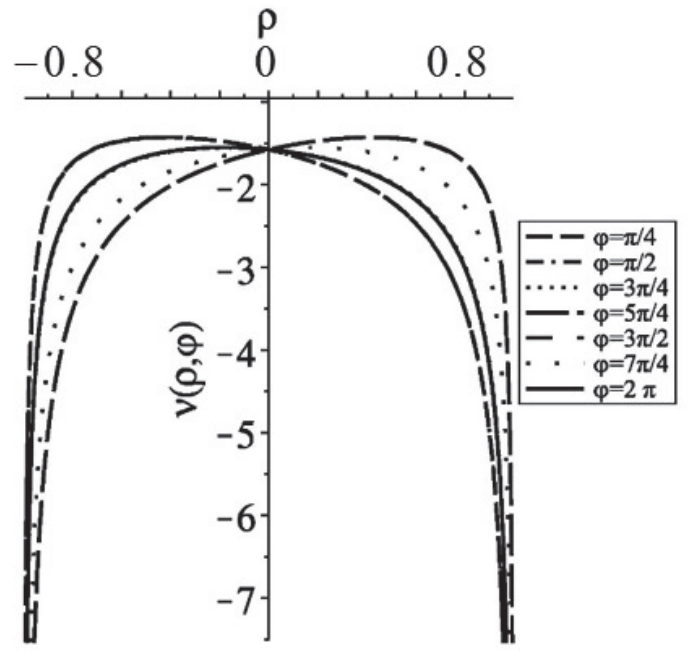

Figure 3: $P_{0}=0, q_{0}=70$

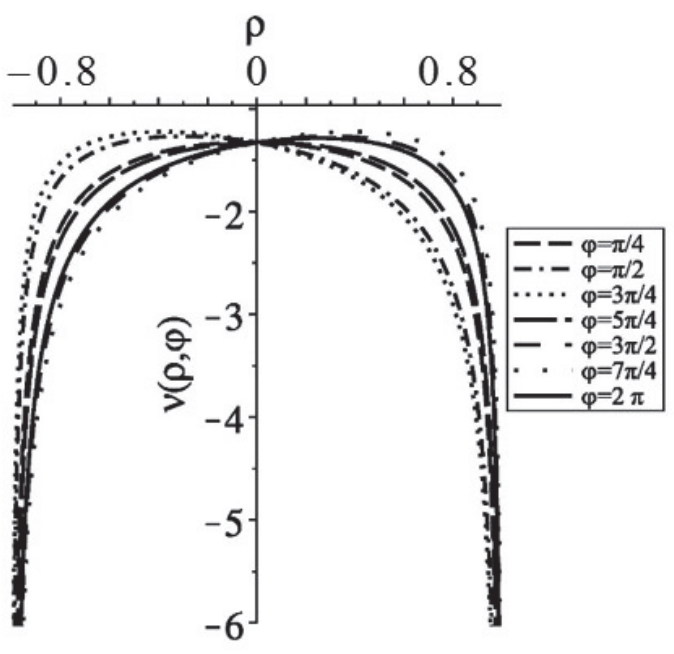

Figure 5: $P_{0}=0.5, q_{0}=70$ 


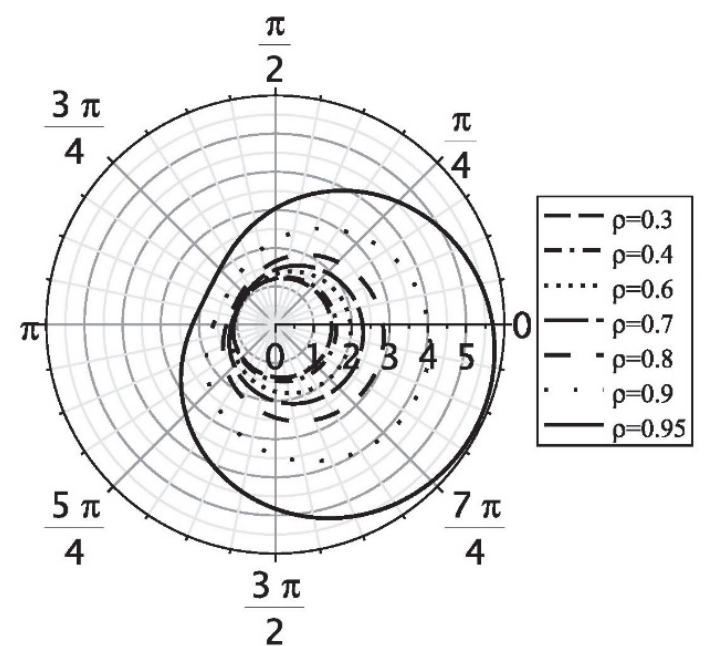

Figure 6: $P_{0}=0.7, q_{0}=70$

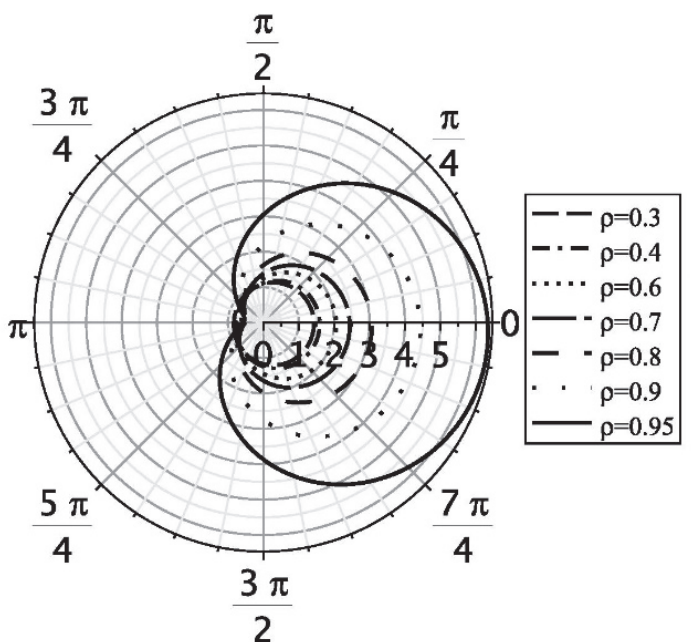

Figure 8: $P_{0}=1, q_{0}=70$

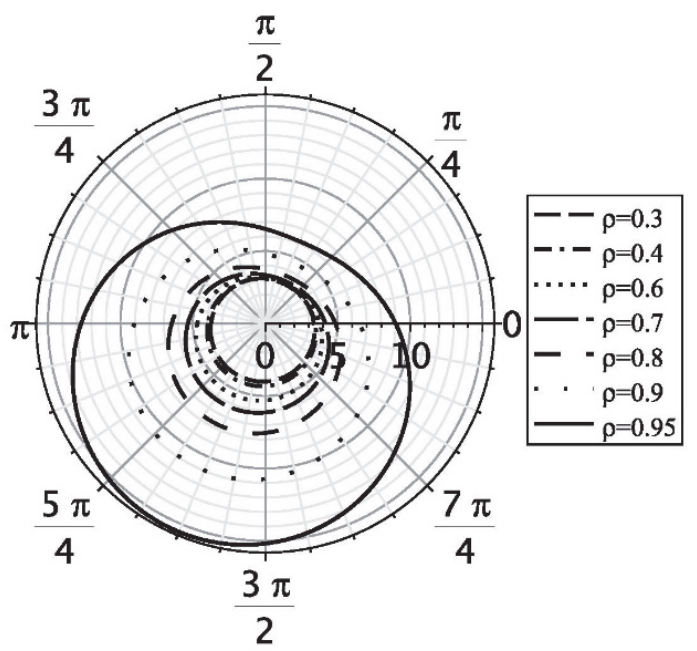

Figure 10: $P_{0}=0, q_{0}=150$

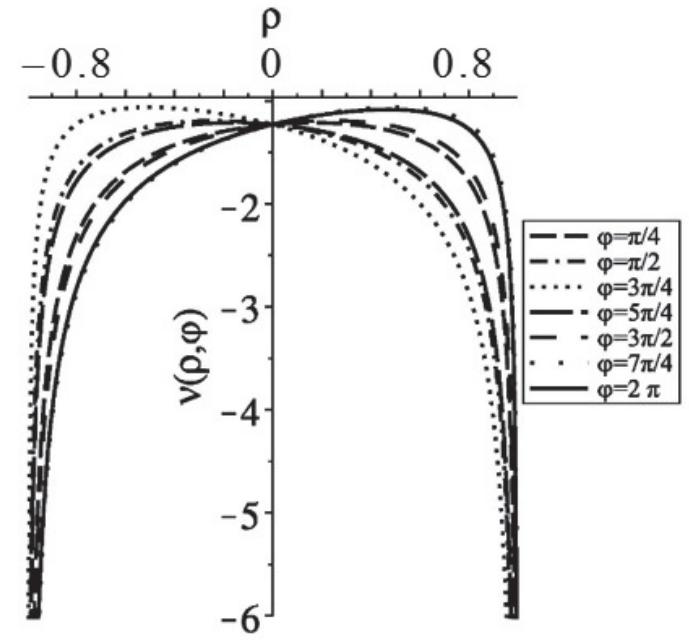

Figure $7: P_{0}=0.7, q_{0}=70$

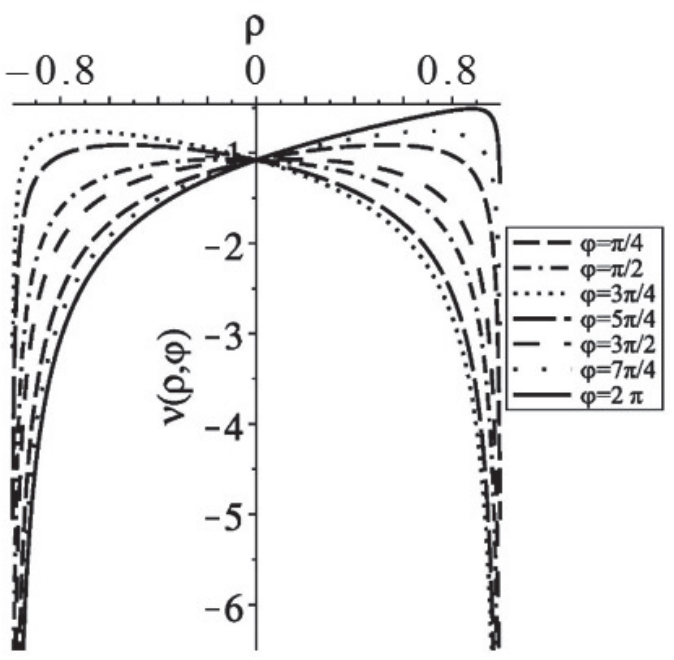

Figure 9: $P_{0}=1, q_{0}=70$

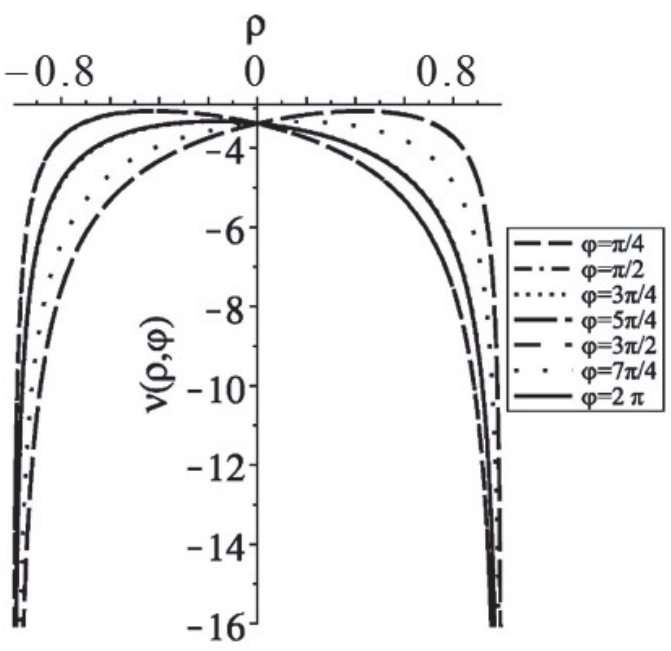

Figure 11: $P_{0}=0, q_{0}=150$ 


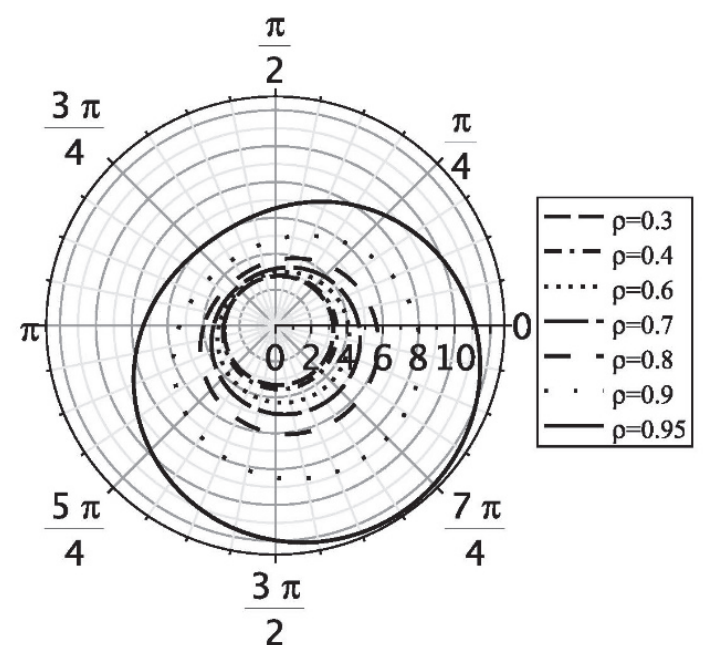

Figure 12: $P_{0}=1, q_{0}=150$

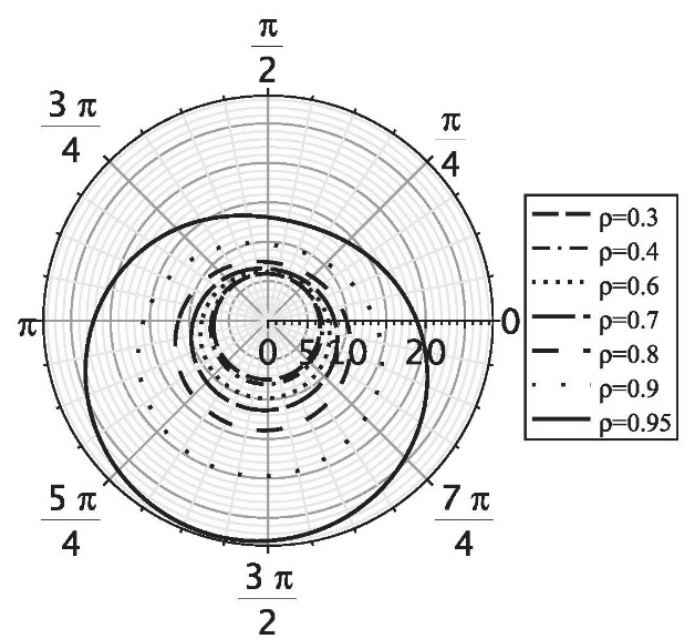

Figure 14: $P_{0}=0.5, q_{0}=70$

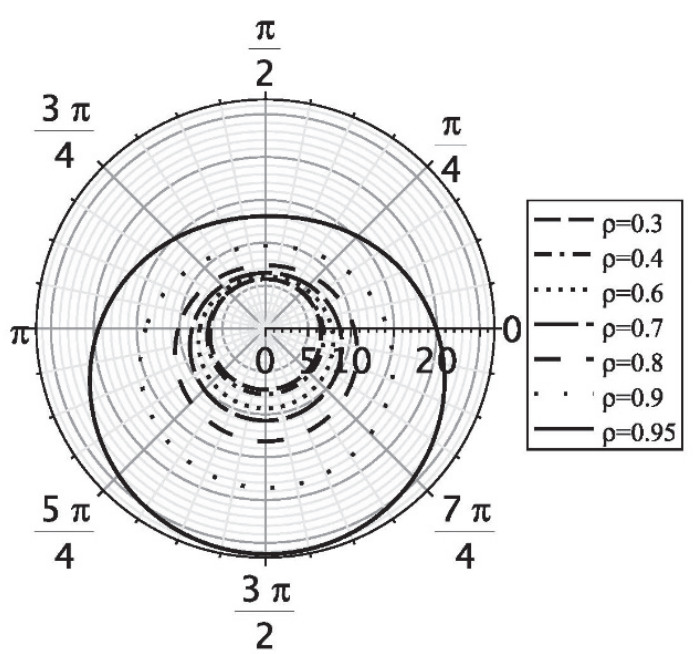

Figure 16: $P_{0}=1, q_{0}=70$

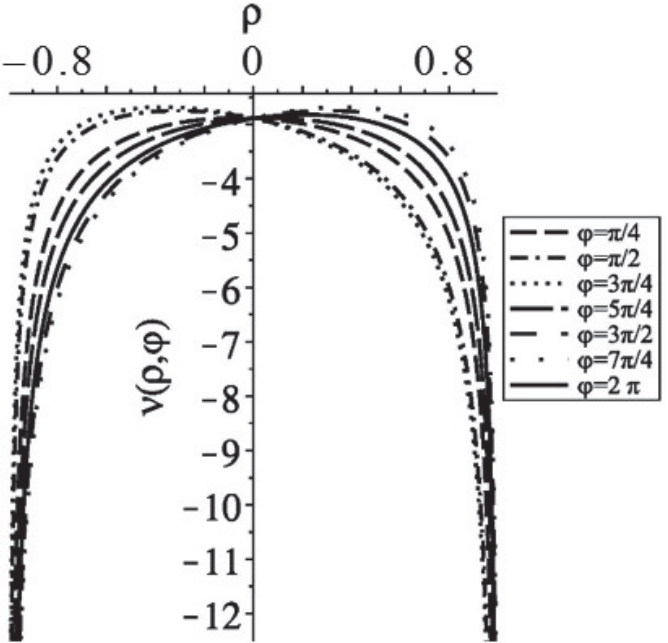

Figure 13: $P_{0}=1, q_{0}=150$

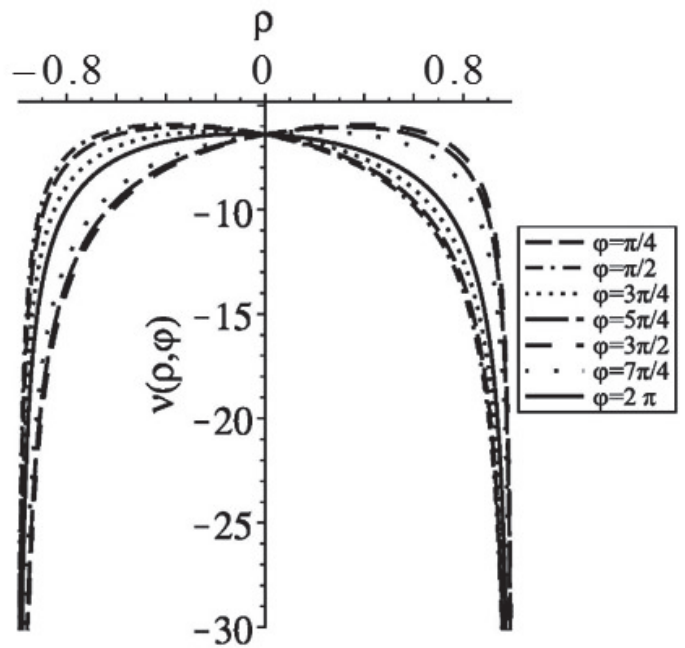

Figure 15: $P_{0}=0.5, q_{0}=70$

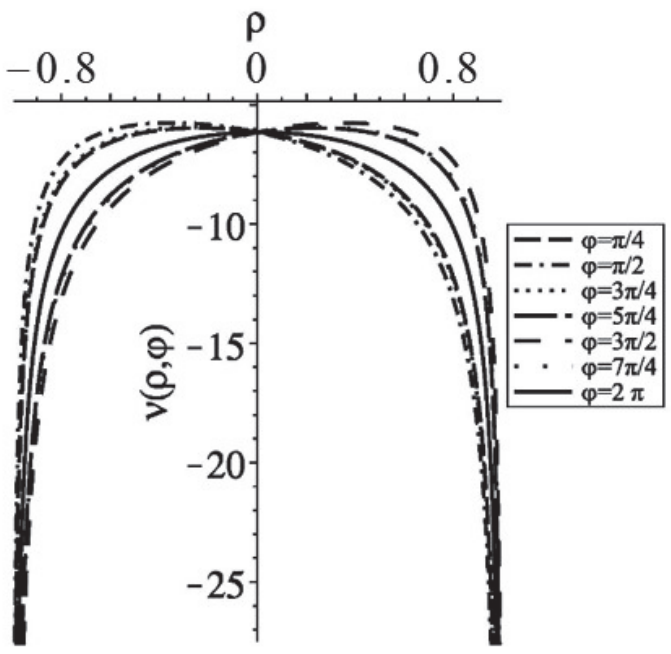

Figure 17: $P_{0}=1, q_{0}=70$ 


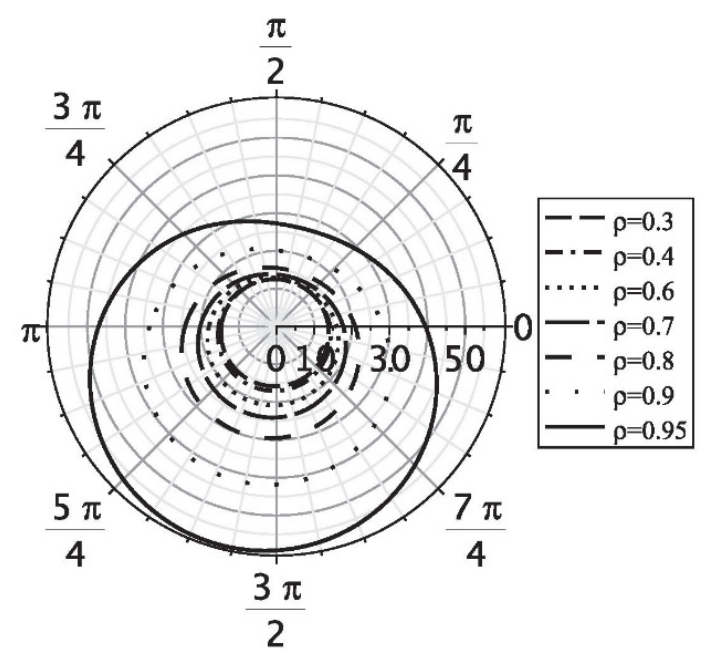

Figure 18: $P_{0}=1, q_{0}=150$

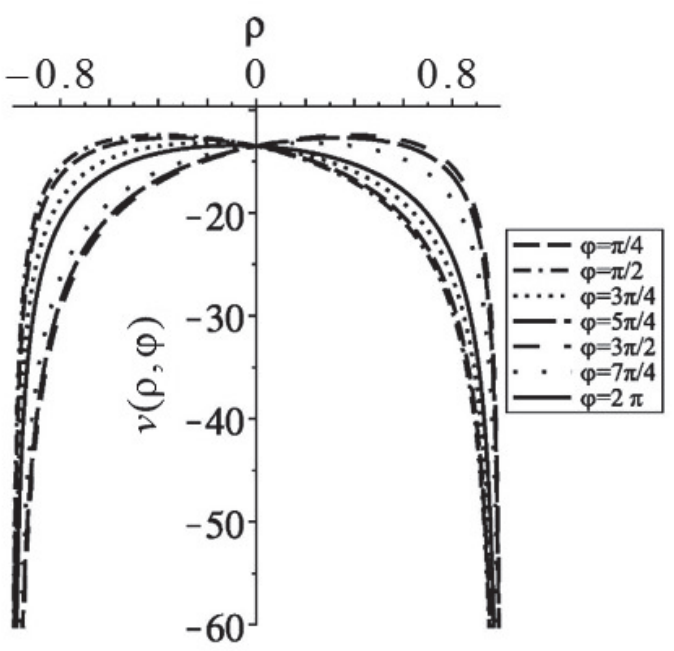

Figure 19: $P_{0}=1, q_{0}=150$

The purpose of the calculations was to establish the influence on the distribution of normal stresses in the neighborhood of the inclusion of the force and temperature fields, as well as the inhomogeneity of the matrix.. The degree of heterogeneity of the matrix in the direction perpendicular to the inclusion, the coefficient are determines: $\zeta_{z}=c_{33}^{+} / c_{33}^{-}$, which characterizes the difference between the elastic properties of half-spaces in the direction of the axis Z. So, for the combination of materials $\mathrm{m} 1-\mathrm{m} 2$, the value $\zeta_{z}=0.7483$, for the combination $\mathrm{m} 3-\mathrm{m} 4$, the value $\zeta_{z}=8.2981$, i.e., the elastic properties of the half spaces in the z-axis direction, for the second combination $\mathrm{m} 3-\mathrm{m} 4$ are much more different than for the first $\mathrm{m} 1-\mathrm{m} 2$.

It has been established (see Figs. 2, 3, and 10,11) that in the absence of force action with the growth of thermal radiation, an increase in stress and a change in the nature of its distribution are observed, which is obviously due to the inhomogeneity of the medium. With fixed thermal radiation and growthing of the force loading for the first combination: $\zeta_{z}=0.7483$, (see Figs. 2, 4, 6, 8 and 3, 5, 7,9) a significant change in the direction of the gradient of normal stresses are observed, while for the second combination, $\zeta_{z}=8.2981$ (Figs. 14, 16 and 15,17) such changes are less pronounced.

Obviously, this regularity is explained by a much greater rigidity (almost an order) of the material of the upper half-space for the second combination, which leads to a more stable direction of increase in stresses. However, in this case, the opposite quantitative picture of the stress change is observed (see Figs. 12, 13 and 18, 19): for the second combination of materials, the normal stresses are four times higher for the same values of the parameters of the force and heat exposure. Note that the regularities found could be identified due to the general non-axisymmetric formulation of the problem.

\section{CONCLUSIONS}

A

$\mathrm{n}$ accurate solution to the non-axisymmetric problem of circular heat radiating inclusion at arbitrary loading, which is in smooth contact with different transversely isotropic spaces, has been designed. The latter allowed, in particular, to investigate the features of the field of normal stresses and their distribution around the inclusion. The resulting expressions for translational $\delta_{z}$ and circular movements $\varphi_{x}, \varphi_{y}$, show that the translational movements depends on the resulting stress $P_{z}$ and the amount of heat flux, and the circular movements $\varphi_{x}, \varphi_{y}$ respective to their resulting moments $M_{x}, M_{y}$, as well as the heat flux.

The proposed approach allows one to obtain the exact solutions of nonaxisymmetric problems of stationary thermoelasticity for interphase inclusions for other types of contact interaction (perfect contact, delamination, etc.) with various transversely isotropic half-spaces.

The existence of such solutions makes it possible not only to identify critical loads and calculate structural elements for strength, but also expand the theoretical base for the application of technical remote monitoring methods for the state 
of various structures for long-term operation [3]. In particular, methods of thermographic and acoustic control, based on knowledge of the distribution of stress and temperature gradients around various internal defects (cracks, inclusions for different types of contact interaction with the medium). These methods form the foundation of operation of various thermal imagers and flaw detectors [3]; therefore, the proposed solutions make it possible to improve the operation of remote monitoring the state of various structures.

\section{REFERENCES}

[1] Kit, G.S., O. P. Sushko, O.P. (2011). Problems of stationary heat conduction and thermoelasticity for a body with a heat permeable disk-shaped inclusion (crack), J Math Sci. 174(3), pp. 309-319. DOI: 10.1007/s10958-011-0300-3.

[2] Kit, G. S., Sushko, O. P. (2011). Axially symmetric problems of stationary heat conduction and thermoelasticity for a body with thermally active or thermally insulated disk inclusion (crack), J Math Sci. 176(4), pp. 561-577.

DOI: $10.1007 /$ s10958-011-0422-7.

[3] Marukha V.I., Panasyuk V.V., Sylovaniuk V.P. (2009). Injection technologies of damaged long-term operation structures renewal. Volume 12, in Facture mechanic and strength of materials: Reference book (Editor-in-Chief V.V. Panasyuk) Lviv: PH "Spolom”, 262 c.

[4] Sylovanyuk, V.P., Marukha, V.I., Yukhym, R.Y. (2010) Strengthening of concrete as a result of filling of the pores and cavities, Mater Sci 46: pp. 70-75. DOI: 10.1007/s11003-010-9265-5.

[5] Efimov, V.V., Krivoj, A.F., Popov, G.Ya. (1998). Problems on the stress concentration near a circular imperfection in a composite elastic medium, Mechanics solids, 33(2), pp. 35-49.

[6] Li, X.F., Fan, T.Y. (2001). The asymptotic stress field for a rigid circular inclusion at the interface of two bonded dissimilar elastic half-space materials, International Journal of Solids and Structures, 38, pp. 8019-8035. DOI: org/10.1016/j.ijsolstr.2009.01.002/.

[7] Kaczynski, A., Kozlowski, W. (2009). Thermal stresses in an elastic space with a perfectly rigid flat inclusion under perpendicular heat flow, International Journal of Solids and Structures, 46, pp. 1772-1777.

DOI: $10.1016 /$ S0020-7683(01)00010-5.

[8] Kaczynski, A., Monastyrsky, B. (2013). A rigid inclusion in an elastic space under the action of a uniform heat flow in the inclusion plane, International Journal of Solids and Structures, 50(16-17), pp.2631-2640.

DOI: $10.1016 /$ j.ijsolstr.2013.04.012.

[9] Kaczynski, A. (2016). On 3D symmetrical thermoelastic anticrack problems Archives of Mechanics, 68(2), pp. 99-112.

[10] Kaczynski, A. (2014). Thermal stress analysis of a three-dimensional anticrack in a transversely isotropic solid, International Journal of Solids and Structures, 51(13), pp. 2382-2389. DOI: 10.1016/j.ijsolstr.2014.03.004.

[11] Kryvyy O.F. (2011). Singular integral relations and equations for a piecewise homogeneous transversally isotropic space with interphase defects, J Math Sci. 176(4), pp 515--531. DOI: 10.1007/s10958-011-0419-2.

[12] Kryvyy, O.F. (2012). Interface circular inclusion under mixed conditions of interaction with a piecewise homogeneous transversally isotropic space, J Math Sci. 184(1), pp. 101-119. DOI: 10.1007/s10958-012-0856-6.

[13] Kryvyi, O.F. (2012). Interface crack in the inhomogeneous transversely isotropic space, Mater Sci. 47(6), pp. 726-736. DOI: $10.1007 /$ s11003-012-9450-9.

[14] Kryvyi, O.F. (2014). Delaminated Interface Inclusion in a Piecewise Homogeneous Transversely Isotropic Space, Mater Sci. 50(2), pp. 245-253. DOI: 10.1007/s11003-014-9714-7.

[15] Kryvyy, O. (2009). The Discontinuous Solution for the Piece-homogeneous Transversal Isotropic Medium, Operator Theory: Advances and Applications, 191.pp. 387 - 398. DOI: 10.1007/978-3-7643-9921-4_25.

[16] Mikhas'kiv, V.V. \& Stasyuk, B.M. (2015) Elastic State of a Sliding Short Fiber Inclusion in Three-Dimensional Matrix, Int Appl Mech, 51(6), pp. 640-647. DOI: 10.1007/s10778-015-0720-8

[17] Panasyuk, V.V., Stadnyk M. M., Sylovaniuk, V.P. (1986) Концентрация напряжений в трехмерных телах с тонкими вкАючениями. К.: Наук. Аумка.

[18] Krivoi, A.F., Radiollo, M.V. (1984) Specific features of the stress field near inclusions in a composite anisotropic plane, Izv. Akad. Nauk SSSR, Mekh. Tverd. Tela, 3. pp. 84-92.

[19] Popov G.Ya. (1982). The stress concentration near punches, sections, thin inclusions and supports. M.: Nauka.

[20] Selvadurai A.P.S. (1980) Asymmetric displacements of a rigid disc inclusion embedded in a transversely isotropic elastic medium of infinite extent, Int. J. Engng Sci., 18 (7), pp. 979-986, DOI: 10.1016/ 0020-7225(80)90070-1.

[21] Nowacki, W. (1986). Thermoelasticity (2nd ed.). Warszawa: PWN-Polish Scientific Publishers. 25, 373 
[22] Haojiang Ding, Weiqiu Chen, L. Zhang (2006). Elasticity of Transversely Isotropic Materials Published by Springer, P.O. Box 17, 3300 AA Dordrecht, The Netherlands. DOI: 10.1007/1-4020-4034-2.

\section{APPENDIX A: CONSTRUCTION OF A DISCONTINUOUS SOLUTION AND SINGULAR INTEGRAL RELATIONS} FOR A PIECEWISE HOMOGENEOUS TRANSVERSELY ISOTROPIC BODY IN $\mathfrak{I}^{\prime}\left(\mathfrak{R}^{3}\right)$

$\mathrm{R}$ elative to the components of the displacement vector $\mathbf{u}=\left\{u_{j}\right\}^{3}=\left\{v_{j+3}\right\}^{3}$ and temperature $T$ using the DuhamelNeumann relations [21], we obtain, with $z \neq 0$, system of differential equations

$$
D\left[\partial_{1}, \partial_{2}, \partial_{3}\right] \mathbf{u}=\beta T, P\left[\partial_{1}, \partial_{2}, \partial_{3}\right] T=0, z \neq 0
$$

where

$$
\begin{aligned}
& D\left[\partial_{1}, \partial_{2}, \partial_{3}\right]=\left\{L_{k j}\right\}^{3}, P\left[\partial_{1}, \partial_{2}, \partial_{3}\right]=\partial_{3}^{2}+\lambda\left(\partial_{1}^{2}+\partial_{2}^{2}\right), \partial_{1} \equiv \partial / \partial x, \partial_{2} \equiv \partial / \partial y, \partial_{3} \equiv \partial / \partial z \\
& L_{11}=c_{11} \partial_{1}^{2}+c_{66} \partial_{2}^{2}+c_{44} \partial_{3}^{2}, \quad L_{12}=\left(c_{12}+c_{66}\right) \partial_{12}^{2}, L_{13}=\left(c_{44}+c_{13}\right) \partial_{13}^{2}, L_{j k}=L_{k j} \\
& L_{22}=c_{66} \partial_{1}^{2}+c_{11} \partial_{2}^{2}+c_{44} \partial_{3}^{2}, \quad L_{23}=\left(c_{44}+c_{23}\right) \partial_{23}^{2}, L_{33}=c_{55} \partial_{1}^{2}+c_{44} \partial_{2}^{2}+c_{33} \partial_{3}^{2}, \\
& \lambda=\lambda_{1} / \lambda_{3}, c_{k j}=\theta(z) c_{k j}^{+}+\theta(-z) c_{k j}^{-}, \quad \lambda_{j}=\theta(z) \lambda_{j}^{+}+\theta(-z) \lambda_{j}^{-}, c_{23}=c_{13}, c_{55}=c_{44}, c_{22}=c_{11} \\
& \boldsymbol{\beta}=\left\{\beta_{1}, \beta_{2}, \beta_{3}\right\}^{T}, \beta_{j}=\theta(z) \beta_{j}^{+}+\theta(-z) \beta_{j}^{+}, \beta_{1}=\beta_{2}, \alpha_{j}=\theta(z) \alpha_{j}^{+}+\theta(-z) \alpha_{j}^{+}, \\
& -\beta_{1}=c_{11} \alpha_{1}+c_{12} \alpha_{2}+c_{13} \alpha_{3},-\beta_{3}=c_{13} \alpha_{1}+c_{23} \alpha_{2}+c_{33} \alpha_{3},
\end{aligned}
$$

$c_{k j}^{ \pm}$- elastic constants, respectively, of the upper and lower half-spaces.

Other components of the vector $\mathbf{v}$ from (1.2) can be found by the formulas

$$
\begin{aligned}
& v_{1}=c_{13}\left(\partial_{1} u_{1}+\partial_{2} u_{2}\right)+c_{33} \partial_{3} u_{3}+\beta_{3} T, \quad v_{2}=c_{44}\left(\partial_{3} u_{2}+\partial_{2} u_{3}\right), \\
& v_{3}=c_{44}\left(\partial_{1} u_{3}+\partial_{3} u_{1}\right), v_{8}=k_{z} \partial_{3} T
\end{aligned}
$$

Based on the representation of the solutions of Eqns. (A.1) for a homogeneous space [22], the components of the vector $\mathbf{v}$ are

$$
v_{j}=\Phi_{j}\left[\psi_{0}, \psi_{1}, \psi_{2}, \psi_{3}\right], j=\overline{1,8}, z \neq 0
$$

Here we use the notation

$$
\begin{aligned}
& \Phi_{j}\left[\psi_{0}, \psi_{1}, \psi_{2}, \psi_{3}\right]=\theta(z) \Phi_{j}^{+}\left[\psi_{0}, \psi_{1}, \psi_{2}, \psi_{3}\right]+\theta(-z) \Phi_{j}^{-}\left[\psi_{0}, \psi_{1}, \psi_{2}, \psi_{3}\right] \\
& \Phi_{1}^{ \pm}\left[\psi_{0}, \psi_{1}, \psi_{2}, \psi_{3}\right]=\sum_{k=0}^{2} \kappa_{k}^{ \pm} \partial_{3}^{2} \psi_{k}, \Phi_{6}^{ \pm}\left[\psi_{0}, \psi_{1}, \psi_{2}, \psi_{3}\right]=\sum_{k=0}^{2} \eta_{k 1}^{ \pm} \partial_{3} \psi_{k}, \\
& \Phi_{j+1}^{ \pm}\left[\psi_{0}, \psi_{1}, \psi_{2}, \psi_{3}\right]=c_{44}^{ \pm}\left((-1)^{j} \partial_{3 j}^{2} \psi_{3}+\sum_{k=0}^{2} \tilde{\eta}_{k 1}^{ \pm} \partial_{23}^{2} \psi_{k}\right), \tilde{\eta}_{k 1}^{ \pm}=\eta_{k 1}^{ \pm}-1, j=1,2
\end{aligned}
$$




$$
\begin{aligned}
& \Phi_{j+3}^{ \pm}\left[\psi_{0}, \psi_{1}, \psi_{2}, \psi_{3}\right]=(-1)^{j+1} \partial_{3-j} \psi_{3}-\sum_{k=0}^{2} \partial_{j} \psi_{k}, j=1,2 \\
& \Phi_{7}^{ \pm}\left[\psi_{0}, \psi_{1}, \psi_{2}, \psi_{3}\right]=\alpha_{02}^{ \pm} \partial_{3}^{2} \psi_{0}, \Phi_{8}\left[\psi_{0}, \psi_{1}, \psi_{2}, \psi_{3}\right]=\alpha_{02} \partial_{3}^{2} \psi_{0} \kappa_{j 2}=\theta(z) \kappa_{j 2}^{+}+\theta(-z) \kappa_{j 2}^{-}, \\
& \alpha_{j 1}=\theta(z) \alpha_{j 1}^{+}+\theta(-z) \alpha_{j 1}^{-}, j=0,1,2, \\
& \boldsymbol{\kappa}_{j}^{ \pm}=\left(c_{13}^{ \pm}+c_{33}^{ \pm} \xi_{j} \alpha_{j 1}^{ \pm}+\beta_{3} \alpha_{j 2}^{ \pm}\right), \alpha_{j 1}^{ \pm}=\frac{\left(b_{2}^{ \pm} \xi_{j}^{2}-a_{2}^{ \pm}\right)}{b_{1}^{ \pm} \xi_{j}^{2}-a_{1}^{ \pm}}, j=0,1,2 \\
& \alpha_{02}^{ \pm}=\frac{\xi_{0}^{2}\left(c_{33}^{ \pm} c_{44}^{ \pm} \xi_{0}^{2}-\left(c_{11}^{ \pm} c_{33}^{ \pm}-c_{13}^{ \pm}\left(c_{13}^{ \pm}+2 c_{44}^{ \pm}\right)\right)\right)+c_{11}^{ \pm} c_{44}^{ \pm}}{\left(b_{1}^{ \pm} \xi_{0}^{2}-a_{1}^{ \pm}\right)}, \alpha_{12}^{ \pm}=\alpha_{22}^{ \pm}=0, \\
& b_{1}^{ \pm}=-\beta_{3}\left(c_{13}^{ \pm}+c_{44}^{ \pm}\right)+\beta_{1} c_{33}^{ \pm}, b_{2}^{ \pm}=-\beta_{3} c_{44}^{ \pm}, a_{1}^{ \pm}=\beta_{1} c_{44}^{ \pm}, a_{2}^{ \pm}=-\beta_{3} c_{11}^{ \pm}+\beta_{1}\left(c_{13}^{ \pm}+c_{44}^{ \pm}\right), \\
& \left(\xi_{0}^{ \pm}\right)^{2}=k_{1}^{ \pm}\left(k_{3}^{ \pm}\right)^{-1},\left(\xi_{3}^{ \pm}\right)^{2}=c_{66}^{ \pm}\left(c_{44}^{ \pm}\right)^{-1},
\end{aligned}
$$

Constants $\xi_{j}^{ \pm}(j=1,2)$ are solutions of equations

$$
c_{33}^{ \pm} c_{44}^{ \pm}\left(\xi_{j}^{ \pm}\right)^{4}+\left[c_{13}^{ \pm}\left(c_{13}^{ \pm}+2 c_{44}^{ \pm}\right)-c_{11}^{ \pm} c_{33}^{ \pm}\right]\left(\xi_{j}^{ \pm}\right)^{2}+c_{11}^{ \pm} c_{44}^{ \pm}=0
$$

The functions $\psi_{j}, j=0,1,2,3$ satisfy the differential equations:

$$
\begin{gathered}
P_{j}\left[\partial_{1}, \partial_{2}, \partial_{3}\right] \psi_{j}=0, z \neq 0 \\
P_{j}=\theta(z) P_{j}^{+}+\theta(-z) P_{j}^{+}, P_{j}^{ \pm}=\partial_{3}^{2}+\left(\xi_{j}^{ \pm}\right)^{2}\left(\partial_{1}^{2}+\partial_{2}^{2}\right), \xi_{j}^{2}=\theta(z)\left(\xi_{j}^{+}\right)^{2}+\theta(-z)\left(\xi_{j}^{-}\right)^{2} .
\end{gathered}
$$

Following [15] we let's pass in the Eqns. (A.4) to the space of generalized functions $\mathfrak{I}^{\prime}\left(\mathfrak{R}^{3}\right)$, As a result, we obtain boundary value problems for the functions $\psi_{j},(j=0,1,2,3)$

$$
\begin{aligned}
& P_{j}\left[\tilde{\partial}_{1}, \tilde{\partial}_{2}, \tilde{\partial}_{3}\right] \psi_{j}=f_{j}, \quad f_{j}=\sum_{k=0}^{1} \delta^{(k)}(₹)\left\langle\partial_{3}^{1-k} \psi_{j}\right\rangle^{-}, \quad \psi_{j} \in \mathfrak{I}_{2}^{\prime}\left(\mathfrak{R} \mathbb{R}^{3}\right) \\
& \tilde{\Phi}_{j}^{+}\left[\psi_{0}^{+}, \psi_{1}^{+}, \psi_{2}^{+}, \psi_{3}^{+}\right]-\tilde{\Phi}_{j}^{-}\left[\psi_{0}^{-}, \psi_{1}^{-}, \psi_{2}^{-}, \psi_{3}^{-}\right]=\chi_{j}^{-}(x, y), \psi_{j}^{ \pm} \in \mathfrak{I}_{ \pm}^{\prime}\left(\mathbb{R}^{3}\right), j=\overline{1,8},
\end{aligned}
$$

We obtain operators $\tilde{\Phi}_{j}^{ \pm}$by replacing in operators $\Phi_{j}^{ \pm}$ordinary derivatives $\partial_{k}$ with generalized ones $\tilde{\partial}_{k}$. Applying the three-dimensional Fourier transform to Eqns. (A.5) we obtain the Riemann problem in the space $\mathfrak{I}^{\prime}\left(\mathfrak{R}^{3}\right)$, by parameter $\alpha_{3}$ to determine the transformants of the functions $\psi_{j}, j=0,1,2,3$.

$$
p_{j}^{+} \Psi^{+}=-p_{j}^{-} \Psi^{-}+Q_{j}, \quad j=\overline{0,3}
$$


To solve the Riemann problem (A.7) using the method proposed in [13], we obtain

$$
\Psi_{j}^{ \pm}= \pm \sum_{k=0}^{1} \frac{(-i r)^{k-1}\left(\mp \omega_{j}^{\mp}\right)^{k} v_{j k}^{0}\left(\alpha_{1}, \alpha_{2}\right)}{\left(\omega_{j}^{+}+\omega_{j}^{-}\right)\left(\alpha_{3} \pm \omega_{j}^{ \pm} r\right)}
$$

where $v_{j k}^{0}\left(\alpha_{1}, \alpha_{2}\right)-$ unknown functions; $r=\sqrt{\alpha_{1}^{2}+\alpha_{2}^{2}}, \omega_{j}^{ \pm}=i \xi_{j}^{ \pm}, j=0,1,2,3$.

Unknown functions $v_{j k}^{0}$, following [15], are expressed through transformants of jumps of thermoelastic characteristics: $\tilde{\chi}_{j}^{-}\left(\alpha_{1}, \alpha_{2}\right)=\mathrm{F}_{2}\left[\chi_{j}^{-}(x, y)\right]$, which allowed, using formulas (A.3), to obtain such expressions for the transformant $V_{j}^{ \pm}\left(\alpha_{1}, \alpha_{2}, z\right)=\theta( \pm z) F_{2}\left[v_{j}\right]:$

$$
\begin{aligned}
& V_{1}^{ \pm}(z)=\theta( \pm z) \sum_{k=1}^{3} e_{k-1}^{ \pm}\left\{q_{1, k}^{1, \pm} r \chi_{1}^{-}-q_{1, k}^{2, \pm} r^{-1}\left(\left(-i \alpha_{2}\right) \chi_{2}^{-}+\left(-i \alpha_{1}\right) \chi_{3}^{-}\right)+q_{1, k}^{3, \pm}\left(\left(-i \alpha_{1}\right) \chi_{4}^{-}+\left(-i \alpha_{2}\right) \chi_{5}^{-}\right)+\right. \\
& \left.+q_{1, k}^{4, \pm} r \chi_{6}^{-}+q_{1, k}^{5, \pm} \chi_{7}^{-}+q_{1, k}^{6, \pm} r^{-1} \chi_{8}^{-}\right\} \\
& V_{2}^{ \pm}(z)=\theta( \pm z)\left\{-r^{-2} \chi_{2}^{-}\left[\sum_{k=1}^{3}\left(-i \alpha_{2}\right)^{2} e_{k-1}^{ \pm} q_{2, k}^{2, \pm}+\left(-i \alpha_{1}\right)^{2} e_{3}^{ \pm} \tilde{q}_{1,3}^{1, \pm}\right]-\left(-i \alpha_{1}\right)\left(-i \alpha_{2}\right) r^{-2} \chi_{3}^{-}\left[\sum_{k=1}^{3} e_{k-1}^{ \pm} q_{2, k}^{2, \pm}-e_{3}^{ \pm} \tilde{q}_{1,3}^{1, \pm}\right]+\right. \\
& +\left(-i \alpha_{1}\right)\left(-i \alpha_{2}\right) r^{-1} \chi_{4}^{-}\left[\sum_{k=1}^{3} e_{k-1}^{ \pm} q_{2, k}^{3, \pm}+e_{3}^{ \pm} \tilde{q}_{1,3}^{2, \pm}\right]+r^{-1} \chi_{5}^{-}\left[\sum_{k=1}^{3}\left(-i \alpha_{2}\right)^{2} e_{k-1}^{ \pm} q_{2, k}^{3, \pm}-\left(-i \alpha_{1}\right)^{2} e_{3}^{ \pm} \tilde{q}_{1,3}^{2, \pm}\right]+ \\
& \left.+\sum_{k=1}^{3} e_{k-1}^{ \pm}\left[q_{2, k}^{1, \pm}\left(-i \alpha_{2}\right) r^{-1} \chi_{1}^{-}+q_{2, k}^{4, \pm}\left(-i \alpha_{2}\right) \chi_{6}^{-}+q_{2, k}^{5, \pm}\left(-i \alpha_{2}\right) r^{-1} \chi_{7}^{-}+q_{2, k}^{6, \pm}\left(-i \alpha_{2}\right) r^{-2} \chi_{8}^{-}\right]\right\} \\
& V_{4}^{ \pm}(z)=\theta( \pm z)\left\{\left(-i \alpha_{2}\right)\left(-i \alpha_{1}\right) r^{-3} \chi_{2}^{-}\left[\sum_{k=1}^{3} e_{k-1}^{ \pm} q_{3, k}^{2, \pm}+e_{3}^{ \pm} \tilde{q}_{2,3}^{1, \pm}\right]+r^{-3} \chi_{3}^{-}\left[\left(-i \alpha_{1}\right)^{2} \sum_{k=1}^{3} e_{k-1}^{ \pm} q_{3, k}^{2, \pm}-e_{3}^{ \pm}\left(-i \alpha_{2}\right)^{2} \tilde{q}_{2,3}^{1, \pm}\right]-\right. \\
& -r^{-2} \chi_{4}^{-}\left[\left(-i \alpha_{1}\right)^{2} \sum_{k=1}^{3} e_{k-1}^{ \pm} q_{3, k}^{3, \pm}+\left(-i \alpha_{2}\right)^{2} e_{3}^{ \pm} \tilde{q}_{2,3}^{2, \pm}\right]-\left(-i \alpha_{2}\right)\left(-i \alpha_{1}\right) r^{-2} \chi_{5}^{-}\left[\sum_{k=1}^{3} e_{k-1}^{ \pm} q_{3, k}^{3, \pm}-e_{3}^{ \pm} \tilde{q}_{2,3}^{2, \pm}\right]- \\
& \left.-\sum_{k=1}^{3} e_{k-1}^{ \pm}\left[q_{3, k}^{1, \pm}\left(-i \alpha_{1}\right) r^{-2} \chi_{1}^{-}+q_{3, k}^{4, \pm}\left(-i \alpha_{1}\right) r^{-1} \chi_{6}^{-}+q_{3, k}^{5, \pm}\left(-i \alpha_{1}\right) r^{-2} \chi_{7}^{-}+q_{3, k}^{6, \pm}\left(-i \alpha_{1}\right) r^{-3} \chi_{8}^{-}\right]\right\} \\
& V_{6}^{ \pm}(\text {z })=\theta( \pm ₹) \sum_{k=1}^{3} e_{k-1}^{ \pm}\left\{q_{4, k}^{1, \pm} r^{-1} \chi_{1}^{-}-\left(\left(-i \alpha_{2}\right) r^{-2} \chi_{2}^{-}+\left(-i \alpha_{1}\right) r^{-2} \chi_{3}^{-}\right) q_{4, k}^{2, \pm}+\right. \\
& \left.+\left(\left(-i \alpha_{1}\right) r^{-1} \chi_{4}^{-}+\left(-i \alpha_{2}\right) r^{-1} \chi_{5}^{-}\right) q_{4, k}^{3, \pm}+q_{4, k}^{4, \pm} \chi_{6}^{-}+q_{4, k}^{5, \pm} r^{-1} \chi_{7}^{-} q_{4, k}^{6, \pm} r^{-2} \chi_{8}^{-}\right\} \\
& V_{7}^{ \pm}(z)=\theta( \pm z) \sum_{k=1}^{3} e_{k-1}^{ \pm}\left\{\chi_{7}^{-} q_{5, k}^{5}+r^{-1} \chi_{8}^{-} q_{5, k}^{6}\right\}, V_{8}^{ \pm}(z)=\theta( \pm z) \sum_{k=1}^{3} e_{k-1}^{ \pm}\left\{r \chi_{7}^{-} q_{6, k}^{5}+\chi_{8}^{-} q_{6, k}^{6}\right\}
\end{aligned}
$$

where

$$
e_{k}^{ \pm}=e^{ \pm i \omega_{k}^{ \pm} r}, \tilde{\mathbf{S}}=\tilde{\mathbf{S}}^{+}-\tilde{\mathbf{S}}^{-}, \tilde{\mathbf{S}}^{ \pm}=\left\{\tilde{s}_{j k}^{ \pm}\right\}=\left(\begin{array}{cc}
\gamma_{30}^{2, \pm} & \gamma_{31}^{2, \pm} \\
\beta_{30}^{1, \pm} & \beta_{31}^{1, \pm}
\end{array}\right), \tilde{\mathbf{S}}^{-1}=\tilde{\mathbf{S}}^{*}=\left\{\tilde{s}_{j k}^{*}\right\}_{j, k=1,2}=\left(\begin{array}{cc}
\tilde{s}_{11}^{*} & \tilde{s}_{12}^{*} \\
\tilde{s}_{21}^{*} & \tilde{s}_{22}^{*}
\end{array}\right) \text {, }
$$




$$
\begin{aligned}
& \mathbf{S}=\mathbf{S}^{+}-\mathbf{S}^{-}, \mathbf{S}^{ \pm}=\left\{s_{j k}^{ \pm}\right\}=\left(\begin{array}{llllll}
\gamma_{00}^{1, \pm} & \gamma_{01}^{1, \pm} & \gamma_{10}^{1, \pm} & \gamma_{11}^{1, \pm} & \gamma_{20}^{1, \pm} & \gamma_{21}^{1, \pm} \\
\gamma_{00}^{2, \pm} & \gamma_{01}^{2, \pm} & \gamma_{10}^{2, \pm} & \gamma_{11}^{2, \pm} & \gamma_{20}^{2, \pm} & \gamma_{21}^{2, \pm} \\
\beta_{00}^{1, \pm} & \beta_{01}^{1, \pm} & \beta_{10}^{1, \pm} & \beta_{11}^{1, \pm} & \beta_{20}^{1, \pm} & \beta_{21}^{1, \pm} \\
\beta_{00}^{2, \pm} & \beta_{01}^{2, \pm} & \beta_{10}^{2, \pm} & \beta_{11}^{2, \pm} & \beta_{20}^{2, \pm} & \beta_{21}^{2, \pm} \\
\beta_{00}^{3, \pm} & \beta_{01}^{3, \pm} & \beta_{10}^{3, \pm} & \beta_{11}^{3, \pm} & \beta_{20}^{3, \pm} & \beta_{21}^{3, \pm} \\
\gamma_{00}^{3, \pm} & \gamma_{01}^{3, \pm} & \gamma_{10}^{3, \pm} & \gamma_{11}^{3, \pm} & \gamma_{20}^{3, \pm} & \gamma_{21}^{3, \pm}
\end{array}\right), \mathbf{S}^{-1}=\mathbf{S}^{*}=\left\{s_{j k}^{*}\right\}, \\
& \beta_{j k}^{1, \pm}=\frac{\left( \pm i \omega_{j}^{\mp}\right)^{k}}{\omega_{j}^{+}+\omega_{j}^{-}}, \beta_{j k}^{2, \pm}=\frac{\left( \pm i \omega_{j}^{ \pm}\right) \alpha_{j 1}^{ \pm}\left( \pm i \omega_{j}^{\mp}\right)^{k}}{\left(\omega_{j}^{+}+\omega_{j}^{-}\right)}, \beta_{j k}^{3, \pm}=\frac{\left(\xi_{j}^{ \pm}\right)^{2} \alpha_{j 2}^{ \pm}\left( \pm i \omega_{j}^{\mp}\right)^{k}}{\left(\omega_{j}^{+}+\omega_{j}^{-}\right)}, \gamma_{3 k}^{2, \pm}=c_{44}^{ \pm} \beta_{3 k}^{1, \pm}\left( \pm i \omega_{k}^{ \pm}\right), \\
& \gamma_{j k}^{1, \pm}=c_{13}^{ \pm} \beta_{j k}^{1, \pm}+c_{33}^{ \pm}\left( \pm i \omega_{j}^{ \pm}\right) \beta_{j k}^{2, \pm}+\beta_{3}^{ \pm} \beta_{j k}^{3, \pm}, \gamma_{j k}^{2, \pm}=c_{44}^{ \pm}\left[\beta_{j k}^{2, \pm}-\beta_{j k}^{1, \pm}\left(\mp \xi_{j}^{ \pm}\right)\right], \gamma_{j k}^{3, \pm}=\left(\mp i \lambda_{3}^{ \pm} \xi_{j}^{ \pm}\right) \beta_{j k}^{3, \pm}, \\
& q_{1, k}^{p, \pm}=\sum_{n=1}^{2} s_{k n}^{ \pm} s_{n p}^{*}, q_{2, k}^{p, \pm}=\sum_{n=3}^{4} s_{k n}^{ \pm} s_{n p}^{*}, q_{3, k}^{p, \pm}=\sum_{n=5}^{6} s_{k n}^{ \pm} s_{n p}^{*}, \tilde{q}_{3, k}^{p, \pm}=\sum_{n=1}^{2} \tilde{s}_{k n}^{ \pm} \tilde{s}_{n p}^{*}
\end{aligned}
$$

The formulas for the definition $V_{3}^{ \pm}\left(\right.$z) and $V_{5}^{ \pm}(z)$ we obtain respectively from the formulas for $V_{3}^{ \pm}(z)$ and $V_{5}^{ \pm}(z)$, by permutations $\chi_{2}^{-} \Leftrightarrow \chi_{3}^{-}, \chi_{4}^{-} \Leftrightarrow \chi_{5}^{-}, \alpha_{1} \Leftrightarrow \alpha_{2}$.

Using expressions (A.9), we compose the transformants of the sums of limiting values: $z= \pm 0$ thermoelastic characteristics of the space, and proceed to the originals. As a result, we obtain singular integral relations in $\mathfrak{I}^{\prime}\left(\mathfrak{R}^{3}\right)$, connecting jumps and the sum of the components of the stress tensor, displacement vectors, temperature and heat flux in the plane:

$$
\begin{aligned}
\chi_{1}^{+}(x, y)= & q_{11} \chi_{1}^{-}+q_{13} \sum_{j=4}^{5} \partial_{j-3} \chi_{j}^{-}+q_{15} \chi_{7}^{-}-\frac{1}{2 \pi} \iint_{\Omega}\left\{q_{12}\left[\chi_{2}^{-} \partial_{2} \frac{1}{r_{0}}+\chi_{3}^{-} \partial_{1} \frac{1}{r_{0}}\right]+q_{14} \chi_{6}^{-} \frac{1}{r_{0}^{3}}-q_{16} \chi_{8}^{-} \frac{1}{r_{0}}\right\} d t d \tau, \\
\chi_{2}^{+}(x, y)= & q_{22}^{+} \chi_{2}^{-}+q_{24} \partial_{2} \chi_{6}^{-}+\frac{1}{2 \pi} \iint_{\Omega}\left\{q_{21} \chi_{1}^{-} \partial_{2} \frac{1}{r_{0}}-\chi_{2}^{-}\left[q_{22} \partial_{1} \frac{x-t}{r_{0}^{2}}+\tilde{q}_{11} \partial_{2} \frac{y-\tau}{r_{0}^{2}}\right]+q_{22}^{-} \chi_{3}^{-} \partial_{2} \frac{x-t}{r_{0}^{2}}+\right. \\
& \left.+q_{23}^{-} \chi_{4}^{-} \partial_{12}^{2} \frac{1}{r_{0}}+\chi_{5}^{-}\left[q_{23} \partial_{2}^{2} \frac{1}{r_{0}}-\tilde{q}_{12} \partial_{1}^{2} \frac{1}{r_{0}}\right]+q_{25} \chi_{7}^{-} \partial_{2} \frac{1}{r_{0}}-q_{26} \chi_{8}^{-} \frac{y-\tau}{r_{0}^{2}}\right\} d t d \tau, \\
\chi_{3}^{+}(x, y)= & q_{22}^{+} \chi_{3}^{-}+q_{24} \partial_{1} \chi_{6}^{-}+\frac{1}{2 \pi} \iint_{\Omega}\left\{q_{22}^{-} \chi_{2}^{-} \partial_{1} \frac{y-\tau}{r_{0}^{2}}-\chi_{3}^{-}\left[q_{22} \partial_{2} \frac{y-\tau}{r_{0}^{2}}+\tilde{q}_{11} \partial_{1} \frac{x-t}{r_{0}^{2}}\right]+\right. \\
& \left.+\chi_{4}^{-}\left[q_{23} \partial_{1}^{2} \frac{1}{r_{0}}-\tilde{q}_{12} \partial_{2}^{2} \frac{1}{r_{0}}\right]++q_{21} \chi_{1}^{-} \partial_{1} \frac{1}{r_{0}}+q_{23}^{-} \chi_{5}^{-} \partial_{12}^{2} \frac{1}{r_{0}}+q_{25} \chi_{7}^{-} \partial_{1} \frac{1}{r_{0}}-q_{26} \chi_{8}^{-} \frac{x-t}{r_{0}^{2}}\right\} d t d \tau, \\
\chi_{4}^{+}(x, y)= & \frac{1}{2 \pi} \iint_{\Omega}\left\{q_{31} \chi_{1}^{-} \frac{x-t}{r_{0}^{2}}-q_{32}^{+} \chi_{2}^{-} \partial_{2} \frac{y-\tau}{r_{0}}-\chi_{3}^{-}\left[q_{32} \partial_{1} \frac{x-t}{r_{0}}-\tilde{q}_{21} \partial_{2} \frac{y-\tau}{r_{0}}\right]-q_{34} \chi_{6}^{-} \partial_{1} \frac{1}{r_{0}}-\right. \\
& \left.-\chi_{4}^{-}\left[q_{33} \partial_{2} \frac{y-\tau}{r_{0}^{2}}+\tilde{q}_{22} \partial_{1} \frac{x-t}{r_{0}^{2}}\right]+q_{33}^{-} \chi_{5}^{-} \partial_{2} \frac{x-t}{r_{0}^{2}}+q_{35} \chi_{7}^{-} \frac{x-t}{r_{0}^{2}}+q_{36} \chi_{8}^{-} \frac{x-t}{r_{0}}\right\} d t d \tau+q_{33}^{+} \chi_{4}^{-},
\end{aligned}
$$




$$
\begin{aligned}
\chi_{5}^{+}(x, y)= & \frac{1}{2 \pi} \iint_{\Omega}\left\{q_{31} \chi_{1}^{-} \frac{y-\tau}{r_{0}^{2}}-\chi_{2}^{-}\left[q_{32} \partial_{2} \frac{y-\tau}{r_{0}}-\tilde{q}_{21} \partial_{1} \frac{x-t}{r_{0}}\right]-q_{32}^{+} \chi_{3}^{-} \partial_{1} \frac{y-\tau}{r_{0}}+q_{33}^{-} \chi_{4}^{-} \partial_{1} \frac{y-\tau}{r_{0}^{2}}-\right. \\
& \left.-\chi_{5}^{-}\left[q_{33} \partial_{1} \frac{x-t}{r_{0}^{2}}+\tilde{q}_{22} \partial_{2} \frac{y-\tau}{r_{0}^{2}}\right]-q_{34} \chi_{6}^{-} \partial_{2} \frac{1}{r_{0}}+q_{35} \chi_{7}^{-} \frac{y-\tau}{r_{0}^{2}}+q_{36} \chi_{8}^{-} \frac{y-\tau}{r_{0}}\right\} d \xi_{1} d \xi_{2}+q_{33}^{+} \chi_{5}^{-}, \\
\chi_{6}^{+}(x, y)= & q_{44} \chi_{6}^{-}+\frac{1}{2 \pi} \iint_{\Omega}\left\{q_{41} \chi_{1}^{-} \frac{1}{r_{0}}+q_{42}\left[\chi_{2}^{-} \frac{y-\tau}{r_{0}^{2}}+\chi_{3}^{-} \frac{x-t}{r_{0}^{2}}\right]+q_{43}\left[\chi_{4}^{-} \partial_{1} \frac{1}{r_{0}}+\chi_{5}^{-} \partial_{2} \frac{1}{r_{0}}\right]+\right. \\
& \left.+q_{45} \chi_{7}^{-} \frac{1}{r_{0}}+q_{46} \chi_{8}^{-} \ln \left(r_{0}\right)\right\} d t d \tau, \\
\chi_{7}^{+}(x, y)= & q_{55} \chi_{7}^{-}+\frac{q_{56}}{2 \pi} \iint_{\Omega} \chi_{8}^{-} \frac{1}{r_{0}} d t d \tau, \chi_{8}^{+}(x, y)=q_{66} \chi_{8}^{-}-\frac{q_{65}}{2 \pi} \iint_{\Omega} \chi_{7}^{-} \frac{1}{r_{0}^{3}} d t d \tau,
\end{aligned}
$$

where

$$
\begin{aligned}
& r_{0}=\sqrt{(x-t)^{2}+(y-\tau)^{2}}, q_{k p}^{ \pm}=\sum_{n=1}^{6} s_{k n}^{ \pm} s_{n p}^{*}, q_{k p}=q_{k p}^{+}+q_{k p}^{-}, \tilde{q}_{k p}=\tilde{q}_{k, 3}^{p,+}+\tilde{q}_{k, 3}^{p,-}, \\
& q_{22}^{ \pm}=q_{22} \pm \tilde{q}_{11}, q_{23}^{ \pm}=q_{23} \pm \tilde{q}_{12}, q_{32}^{ \pm}=q_{32} \pm \tilde{q}_{21}, q_{33}^{ \pm}=q_{33} \pm \tilde{q}_{22} .
\end{aligned}
$$

Differentiation operators in space $\mathfrak{I}^{\prime}\left(\mathfrak{R}^{3}\right)$, have readily verifiable properties

$$
\begin{aligned}
& \partial_{2} \frac{x-t}{r_{0}^{2}}=\partial_{1} \frac{y-\tau}{r_{0}^{2}}, \partial_{2} \frac{x-t}{r_{0}}=\partial_{1} \frac{y-\tau}{r_{0}}, \partial_{1} \frac{x-t}{r_{0}^{2}}+\partial_{2} \frac{y-\tau}{r_{0}^{2}}=2 \pi \delta(x-t, y-\tau), \\
& \partial_{1}^{2} \frac{1}{r_{0}}+\partial_{2}^{2} \frac{1}{r_{0}}=\frac{1}{r_{0}^{3}}, \partial_{1} \frac{x-t}{r_{0}}+\partial_{2} \frac{y-\tau}{r_{0}}=\frac{1}{r_{0}}, \mathrm{D} \overline{\mathrm{D}} r_{0}=\frac{1}{r_{0}}, \mathrm{D}=\partial_{1}+i \partial_{2} \\
& \mathrm{D} \frac{1}{\vartheta}=\overline{\mathrm{D}} \frac{1}{\bar{\vartheta}}=0, \vartheta=(x-t)+i\left(y-\tau, r_{0}^{2}=\vartheta \bar{\vartheta} .\right.
\end{aligned}
$$

Using properties (A.11), with respect to complex combinations of jumps and sums: $\tau^{ \pm}=\chi_{3}^{ \pm}+i \chi_{2}^{ \pm}, u^{ \pm}=\chi_{4}^{ \pm}+i \chi_{5}^{ \pm}$, ratio (A.10) can be rewritten as

$$
\begin{aligned}
\chi_{1}^{+}= & q_{11} \chi_{1}^{-}+\frac{q_{13}}{2}\left(\mathrm{D} \bar{u}^{-}+\overline{\mathrm{D}} u^{-}\right)+\frac{q_{12}}{2}\left(\mathrm{DK}\left[\bar{\tau}^{-}\right]+\overline{\mathrm{D}} \mathrm{K}\left[\tau^{-}\right]\right)-q_{14} \mathrm{D} \overline{\mathrm{D}} \mathrm{K}\left[\chi_{6}^{-}\right]+q_{15} \chi_{7}^{-}+q_{16} \mathrm{~K}\left[\chi_{8}^{-}\right], \\
\tau^{+}= & \frac{q_{22}^{+}}{2} \tau^{-}-q_{24} \mathrm{D} \chi_{6}^{-}-q_{21} \mathrm{DK}\left[\chi_{1}^{-}\right]+\frac{q_{22}^{-}}{2} \mathrm{D} \overline{\mathrm{K}}_{0}\left[\bar{\tau}^{-}\right]-\frac{q_{23}^{+}}{2} \mathrm{D} \overline{\mathrm{D}} \mathrm{K}\left[u^{-}\right]-\frac{q_{23}^{-}}{2} \mathrm{D}^{2} \mathrm{~K}\left[\bar{u}^{-}\right]- \\
& -q_{25} \mathrm{DK}\left[\chi_{7}^{-}\right]+q_{26} \overline{\mathrm{K}}_{0}\left[\chi_{8}^{-}\right], \\
\bar{\tau}^{+}= & \frac{q_{22}^{+}}{2} \bar{\tau}^{-}-q_{24} \overline{\mathrm{D}} \chi_{6}^{-}-q_{21} \overline{\mathrm{D}} \mathrm{K}\left[\chi_{1}^{-}\right]-\frac{q_{22}^{-}}{2} \overline{\mathrm{D}} \mathrm{K}_{0}\left[\tau^{-}\right]-\frac{q_{23}^{+}}{2} \mathrm{D} \overline{\mathrm{D}}\left[\bar{u}^{-}\right]-\frac{q_{23}^{-}}{2} \overline{\mathrm{D}}^{2} \mathrm{~K}\left[u^{-}\right]- \\
& -q_{25} \overline{\mathrm{D}} \mathrm{K}\left[\chi_{7}^{-}\right]+q_{26} \mathrm{~K}_{0}\left[\chi_{8}^{-}\right],
\end{aligned}
$$




$$
\begin{aligned}
u^{+}= & \frac{q_{33}^{+}}{2} u^{-}+q_{31} \overline{\mathrm{K}}_{0}\left[\chi_{1}^{-}\right]+\frac{q_{32}^{+}}{2} \mathrm{~K}\left[\tau^{-}\right]+\frac{q_{32}^{-}}{2} \mathrm{DK}\left[\vartheta \bar{\tau}^{-}\right]+\frac{q_{33}^{-}}{2} \mathrm{D} \overline{\mathrm{K}}_{0}\left[\bar{u}^{-}\right]-q_{34} \mathrm{DK}\left[\chi_{6}^{-}\right]+ \\
& +q_{35} \overline{\mathrm{K}}_{0}\left[\chi_{7}^{-}\right]+q_{36} \mathrm{~K}\left[\vartheta \chi_{8}^{-}\right], \\
\bar{u}^{+}= & \frac{q_{33}^{+}}{2} \bar{u}^{-}+q_{31} \mathrm{~K}_{0}\left[\chi_{1}^{-}\right]+\frac{q_{32}^{+}}{2} \mathrm{~K}\left[\bar{\tau}^{-}\right]+\frac{q_{32}^{-}}{2} \overline{\mathrm{D}} \mathrm{K}\left[\bar{\vartheta}^{-}\right]+\frac{q_{33}^{-}}{2} \overline{\mathrm{D}} \mathrm{K}_{0}\left[u^{-}\right]-q_{34} \overline{\mathrm{D}} \mathrm{K}\left[\chi_{6}^{-}\right]+ \\
& +q_{35} \mathrm{~K}_{0}\left[\chi_{7}^{-}\right]+q_{36} \mathrm{~K}\left[\bar{\vartheta} \chi_{8}^{-}\right], \\
\chi_{6}^{+}= & q_{44} \chi_{6}^{-}+q_{41} \mathrm{~K}\left[\chi_{1}^{-}\right]-\frac{q_{42}}{2}\left[\mathrm{~K}_{0}\left[\tau^{-}\right]+\overline{\mathrm{K}}_{0}\left[\bar{\tau}^{-}\right]\right]+\frac{q_{43}}{2}\left[\overline{\mathrm{D}} \mathrm{K}\left[u^{-}\right]+\mathrm{DK}\left[\bar{u}^{-}\right]\right]+ \\
& +q_{45} \mathrm{~K}\left[\chi_{7}^{-}\right]+q_{46} \mathrm{~K}_{1}\left[\chi_{8}^{-}\right], \\
\chi_{7}^{+}= & q_{55} \chi_{7}^{-}+q_{56} \mathrm{~K}\left[\chi_{8}^{-}\right], \chi_{8}^{+}=q_{66} \chi_{8}^{-}-q_{65} \mathrm{D} \overline{\mathrm{D}} \mathrm{K}\left[\chi_{7}^{-}\right] .
\end{aligned}
$$

Here we use the notation

$$
\mathrm{K}\left[\chi_{j}^{-}\right]=\frac{1}{2 \pi} \iint_{\Omega} \frac{\chi_{j}^{-}(t, \tau)}{r_{0}} d t d \tau, \mathrm{K}_{0}\left[\chi_{j}^{-}\right]=\frac{1}{2 \pi} \iint_{\Omega} \frac{\chi_{j}^{-}(t, \tau)}{\vartheta} d t d \tau, \mathrm{K}_{1}\left[\chi_{j}^{-}\right]=\frac{1}{2 \pi} \iint_{\Omega} \chi_{j}^{-}(t, \tau) \ln \left(r_{0}\right) d t d \tau .
$$

The obtained SIS (A.9) and (A.11) generalize the SIS obtained in [15], and allow thermoelasticity problems on interfacial defects in a piecewise homogeneous transversely isotropic space to be reduced directly to SIE systems. 